\title{
The Deterministic Generation of Photons by Cavity Quantum Electrodynamics
}

\author{
H. Walther
}

1.1

Introduction

Cavity quantum electrodynamics allows the study of strong coupling between excited atoms and a single mode of a resonant cavity. As a consequence of the strong coupling the cavity field and the atom are entangled. The system thus provides important ingredients necessary for studies of quantum information processing. In addition the periodic exchange of a photon between atom and cavity can be studied. It thus represents the ideal system to investigate the Jaynes-Cummings model and to study a variety of phenomena related to the dynamics of the photon exchange such as collapse and revivals depending on the statistics of the photon field. Furthermore, it is an ideal system to study the quantum measurement process. The system leads to masers and lasers which sustain oscillations with less than one atom on average in the cavity and can, in addition, be used as a deterministic photon source. The setup allows to study in detail the conditions necessary to obtain nonclassical radiation, i.e. radiation with sub-Poissonian photon statistics and even photon number states; this is the case even when Poissonian pumping is used.

This paper reviews the work on cavity quantum electrodynamics performed with the one-atom maser and with a single ion trap laser. We will start with the discussion of the one-atom maser. For a more detailed discussion of those experiments see also [1].

\section{2}

Oscillatory Exchange of Photons Between an Atom and a Cavity Field (Strong Coupling) - the One-atom Maser or Micromaser

The single-mode radiation field of the one-atom maser, or micromaser, is the prototype of an open driven quantum system. It has been successfully used for studies - both experimental and theoretical - of the quantum interaction

Elements of Quantum Information. Edited by Wolfgang P. Schleich and Herbert Walther Copyright (c) 2007 WILEY-VCH Verlag GmbH \& Co. KGaA, Weinheim ISBN: 978-3-527-40725-5 
between two-level Rydberg atoms and one privileged microwave mode of the radiation field.

In a one-atom maser (or laser)For laser see Sect. 1.4.1, simple two-level atoms are strongly coupled to a single mode of the radiation field that is resonant, or nearly resonant, with the atomic transition. This situation is described by the Jaynes-Cummings Hamiltonian [2],

$$
H=\hbar \omega a^{\dagger} a+\hbar(\omega+\Delta) \sigma^{\dagger} \sigma-\hbar g(t)\left(a^{\dagger} \sigma+\sigma^{\dagger} a\right),
$$

where $a, a^{\dagger}$ are the photon annihilation and creation operators, $\sigma^{\dagger}$ and $\sigma$ are the raising and lowering operators of the atomic transition, and $g(t)$ is the time-dependent coupling strength between the atomic transition and the cavity mode. The frequency of the cavity mode is $\omega$, and the atomic transition differs from this by the detuning $\Delta$. The time dependence of $g(t)$ originates in the passage of the atom through the resonator. Typically, the simple form

$$
g(t)=\left\{\begin{array}{cl}
2 \Omega & \text { for } t_{0}<t<t_{0}+t_{\text {int }} \\
0 & \text { otherwise }
\end{array}\right.
$$

describes matters sufficiently well. Here $\Omega$ is the (effective) Rabi frequency, $t_{0}$ is the instant when the atom in question enters the cavity, and $t_{\text {int }}$ is the duration of the interaction.

Although several experiments have been published in which the strong coupling between atoms and a single cavity mode is exploited, the micromaser is the only strongly coupled system in which a steady-state quantum field has been created with single atoms or less simultaneously in the cavity. The first paper on the one-atom maser was published in [3]. The experiment on the realization of the two-photon maser-oscillator [4] which is based on a related scheme as the one-atom maser also produces a steady-state field, however, the average number of atoms at threshold is about five. Excited-state atoms entering the cavity drive the micromaser field, each atom contributing to the quantum cavity field. Above the threshold of maser action, the steady-state field can be strongly non-classical depending on the particular parameters of the system.

The one-atom maser or micromaser was for a long time the only experimental apparatus that distinctly passes the quantum threshold and enters the non-classical regime. (For a detailed discussion see [1]). The single atom laser recently described by Kimble and coworkers [5] also reaches the quantum limit. The quantum threshold strictly only applies to systems with only one atom at a time in the cavity, since collective atom effects act to destroy quantum effects (see, for example, [6]). A detailed comparison of the different micromaser systems is given in [1]. This shows that the very high $Q$ factor of the micromaser cavity is the feature that allows a steady-state field to be generated with properties that can only be described by quantum mechanics. The 
cavity lifetime of the one-atom maser is more than two orders of magnitude larger than in other related experiments [7]. Work in the optical spectral region is being very actively pursued at the moment (see Sect. 1.4). Furthermore, quantum dots embedded in a semi-conductor micro-cavity [8-10] are another important field of activity as well as semiconductor cavity systems [11].

An important feature of the one-atom maser is its ability to generate steadystate sub-Poissonian (and hence non-classical) fields in the cavity. The extreme sub-Poissonian state is a Fock state, or number state. A Fock state is a state with a fixed number of photons and is maximally distant from what is typically known as a classical light field. These states are the quantum mechanical basis states of the electromagnetic field and are used in nearly all aspects of quantum optics. However, the extreme non-classical nature of these states has made them difficult to produce experimentally, and current sources are limited to the production of single photons [12], see also Chap. 1.4. The oneatom maser, with its high $Q$ factor and hence long photon lifetime, is the most suitable experimental apparatus to produce such states, and their production has therefore been a major focus of recent work on this apparatus, whereby evidence of up to 5-photon Fock states could be achieved. In the following we will describe the micromaser in some detail and provide a review of the recent work on the production of Fock states.

\subsection{1}

\section{Experimental Set-up of the One-atom Maser}

The scheme of the experimental set-up of the one-atom maser used in our laboratory is displayed in Fig. 16.4. The shown set-up is more elaborate than the system described in the first publication [3]. The standard set-up of the one-atom maser employs the effusive output of a rubidium oven, which is collimated into two atomic beams: a central one passing directly into a ${ }^{3} \mathrm{He}-$ ${ }^{4} \mathrm{He}$ dilution refrigerator and a second one directed to an auxiliary excitation region. The central beam passes through the superconducting niobium cavity and the state-selective field ionization apparatus housed in the cryostat. The superconducting cavity has a $Q$-factor of up to $4 \times 10^{10}$, which corresponds to an average lifetime of a photon in the cavity of $0.3 \mathrm{~s}$ [13]. The photon lifetime is therefore much longer than the interaction time of an atom with the maser field, being approximately $30-130 \mu$ s for a thermal atomic beam, and therefore the atomic beam is sufficiently weak to make the probability of two atoms being in the cavity at the same time negligible.

The two-level transitions used in the experiments are between ${ }^{85} \mathrm{Rb}$ Rydberg levels. The rubidium atoms are excited by laser excitation into the $63 P_{3 / 2}$ upper maser level. A frequency-doubled dye laser $(\lambda=297 \mathrm{~nm})$ is used to excite the atoms to the Rydberg state from the $5 S_{1 / 2}(F=3)$ ground state. 


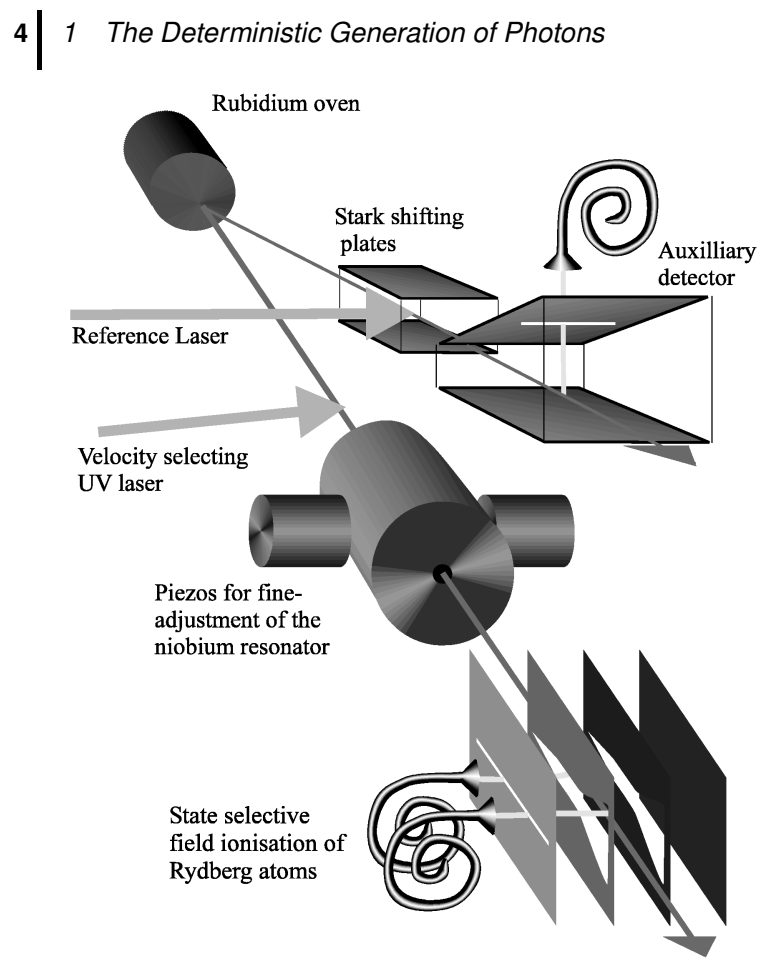

Fig. 1.1 The atoms leaving the rubidium oven are excited into the $63 P_{3 / 2}$ Rydberg state by means of a UV laser at an angle of $11^{\circ}$. After the cavity the atoms are detected by stateselective field ionisation. The cavity is tuned with two piezo translators. An auxiliary atomic beam is used to stabilise the laser frequency.

The laser is locked to a Stark-shifted atomic resonance of the auxiliary beam, thus allowing the velocity subgroup selected by excitation to be continuously changed within the range of the velocity distribution of the atoms. In this way the interaction time of the atoms with the cavity is controlled.

The lower maser level is either the $61 D_{5 / 2}$ or the $61 D_{3 / 2}$ for cavity frequencies of $21.456 \mathrm{GHz}$ and $21.50658 \mathrm{GHz}$, and the vacuum Rabi frequencies of these transitions are $44 \mathrm{kHz}$ and $20 \mathrm{kHz}$, respectively. Surface impurities in the cavity tend to alter the values of these parameters and therefore some calibration is required. In more recent experiments, trapping states (see below) have been used to provide accurate values of the parameters. Velocity selection is provided by angling the excitation laser towards the main atomic beam at $11^{\circ}$ to the normal. The dye laser was locked, by means of an external feedback control [14], to the $5 S_{1 / 2}(F=3)-63 P_{3 / 2}$ transition of the reference atomic beam excited at normal incidence. The exciting laser light from the frequencydoubled laser is linearly polarized and aligned with the linear polarization of the microwave cavity. Only transitions $\Delta m=0$ are thus allowed. This guarantees the two-level nature of the microwave interaction. For a recent paper on this fact see [15]. 
Tuning the laser, which changes the velocity of the beam and thus the interaction time, was performed by Stark shifting the resonance frequency with a stabilized voltage source. This enabled the laser to be tuned while remaining locked to an atomic transition.

Collective effects caused by the entry of more than one atom into the cavity reduce the appearance of quantum effects in the cavity field. An upper limit for the one-atom condition can be arbitrarily considered to be 0.2 atoms on average in the cavity. For a cavity decay time of $0.3 \mathrm{~s}$ this corresponds to a pump rate $N_{\mathrm{ex}}=1500$, whereby $N_{\mathrm{ex}}=R \cdot \tau_{\text {cav }}$ with $R$ for the atomic flux. Above this limit collective effects play an increasingly larger role in the maser dynamics and the detailed predictions of the theory become increasingly inaccurate [6,16-19]. In fact, for some special conditions as in the case of quantum mechanical states (for example, in the case of the trapping states) even this limit is too high. Some care therefore needs to be taken to ensure that these collective effects do not significantly influence the maser field statistics.

\subsection{2}

\section{One-atom Maser as a Source of Non-classical Light}

In the typical operation of the one-atom maser, when collective effects can be ignored and the interaction time is much shorter than the cavity photon lifetime, the strategy to describe the one-atom maser is straightforward. While an atom flies through the cavity the coupled atom field system is described by the Jaynes-Cummings Hamiltonian (equation 1.1), and during the intervals between the successive atoms the evolution of the field is governed by the master equation of a harmonic oscillator damped by a thermal bath. The derivation of the steady-state photon statistics has turned from a research topic $[20,21]$ to a textbook matter (see, for example, $[22,23]$; and [24] is a tutorial on micromaser physics). The problem can be solved analytically, and one obtains the following probability for finding $n$ photons in the cavity when the maser has reached steady state:

$$
P_{n}=P_{0}\left(\frac{n_{\mathrm{th}}}{1+n_{\mathrm{th}}}\right)^{n} \prod_{k=1}^{n}\left(1+\frac{N_{\mathrm{ex}} \mathcal{A}_{k}}{k n_{\mathrm{th}}}\right),
$$

where $\mathcal{A}_{k}=\sin ^{2}\left(\Omega t_{\text {int }} \sqrt{k+1}\right)$ is the emission probability and $n_{\text {th }}$ is the thermal photon number. The normalized pump rate $N_{\mathrm{ex}}$ is the dimensionless parameter that is used in Fig. 1.2 to compare different operating conditions of the one-atom maser. It is given by $N_{\mathrm{ex}}=R \tau_{\text {cav }}$, where $R$ is the atomic rate and $\tau_{\text {cav }}$ is the cavity photon lifetime. The probability of zero photons, $P_{0}$, is determined by normalization to unit total probability, $\sum_{n=0}^{\infty} P_{n}=1$.

With the probabilities $P_{n}$ at hand, one can calculate moments of the photon number, in particular the mean photon number $\langle n\rangle$ and the variance $\left\langle n^{2}\right\rangle-$ 
$\langle n\rangle^{2}$. Following Fano [25] and Mandel [26], we use the normalized variance, the so-called $Q$ parameter,

$$
Q=\frac{\left\langle n^{2}\right\rangle-\langle n\rangle^{2}}{\langle n\rangle}-1,
$$

as a convenient numerical measure of the photon number variance. A Poissonian distribution has $Q=0$; for $Q<0$ one speaks of a sub-Poissonian distribution, and for $Q>0$ one has a super-Poissonian distribution. Fig. 1.2 shows the normalized mean photon number $\left(\langle n\rangle / N_{\mathrm{ex}}\right)$ and the normalized variance $(Q)$ as functions of (top) the interaction time of the atoms with the cavity photons, and (bottom) the pump rate, which are the two experimentally variable parameters.

A parameter that provides a good qualitative comparison of different oneatom maser regimes is the so-called pump parameter, given by $\Theta=\sqrt{N_{\mathrm{ex}}} \Omega t_{\text {int }}$. It is useful for locating features of the maser operating under a wide variety of conditions. For example, one can see in Fig. 1.2 that the maxima of the $Q$ parameter occur when $\Theta$ is (roughly) a multiple of $2 \pi$.

When the value of the pump parameter is $\Theta=1$, the average photon number starts to rise steeply, the photon-statistical parameter $Q$ reaches a local maximum, and the one-photon probability $P_{1}$ becomes larger than the nophoton probability $P_{0}$ for the first time. This point is therefore known as the maser threshold. Another position of interest is where the statistical parameter $Q$ crosses zero for the first time. There the photon distribution passes from a classically shaped super-Poissonian distribution to a sub-Poissonian distribution. This quantum threshold lies at approximately $\Theta=\pi / 2$. At this point nearly all atoms deposit photons in the cavity and the field has a Poissonian distribution with an average value equal to that of the atomic pump rate $N_{\text {ex }}$. Above this point the maser field becomes sub-Poissonian and therefore distinctly quantum mechanical, since the average energy in the cavity field is stabilized by the one-atom maser interaction. Some super-Poissonian peaks appear above this position; these are bistable points in which quantum jumps between two sub-Poissonian distributions occur. The statistics are therefore quantum mechanical in origin, even if they lie in a classical region. The atomic rate, as a function of the interaction time, required to cross the maser threshold and quantum thresholds are indicated in Fig. 1.2 by the lines $R_{\text {th }}$ and $Q_{\text {th }}$, respectively.

The threshold for quantum behavior is really only applicable to the singleatom regime, in which the possibility of collective effects is very low. When collective effects cannot be ignored, the position of this line is somewhat modified. Moreover, while some basic parameters may still apply at this value, the upper bound of collective effects for more exotic states such as trapping states 

pump parameter $(\Theta / \pi)$

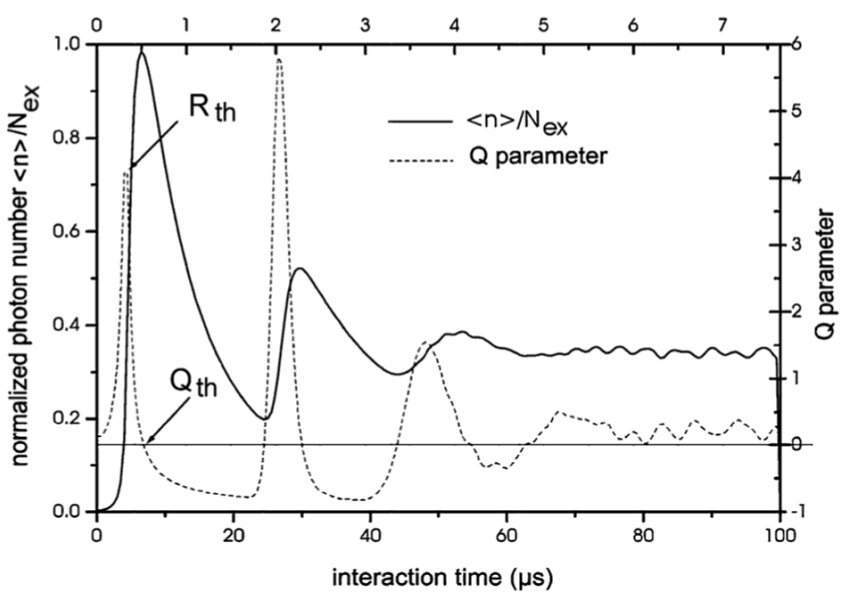

pump parameter $(\Theta / \pi)$

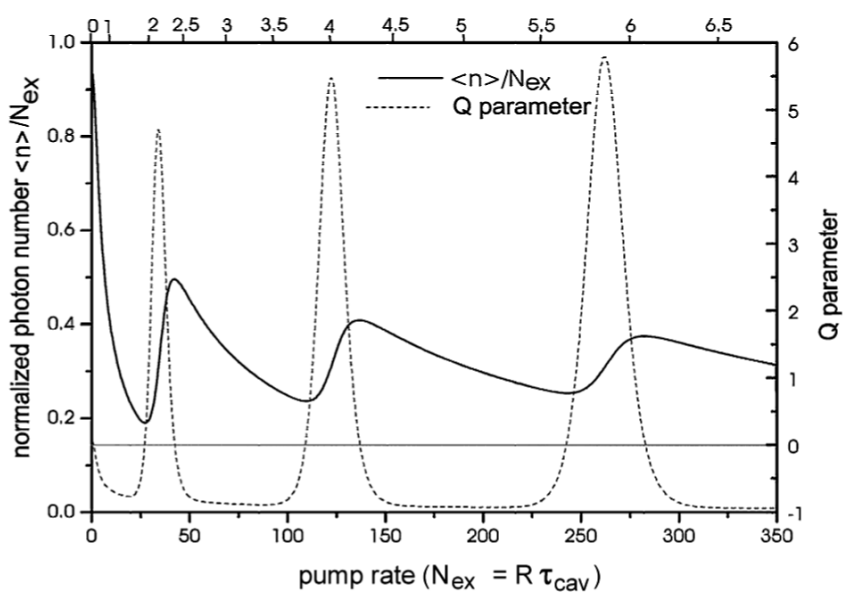

Fig. 1.2 The pump curve of the one-atom maser as obtained from equation (1.3). In both plots, the temperature is set at $0.5 \mathrm{~K}$, which corresponds to $n_{\mathrm{th}}=0.1$ thermal photons. Top: The normalized photon number as a function of the interaction time (bottom axis), for a pump rate $N_{\mathrm{ex}}=40$. Bottom: The normalized photon number as a function of the pump rate $N_{\mathrm{ex}}$ (bottom axis), for an

interaction time $t_{\text {int }}=30 \mu$ s. The top axis of both plots shows the equivalent value of the pump parameter, $\Theta$, for these conditions (see text). The thresholds of maser action $\left(R_{\text {th }}\right)$ and quantum field statistics $\left(Q_{\text {th }}\right)$ are indicated. Note that the micromaser exhibits thresholdless behavior for the parameters of the bottom plot.

is considerably lower since they are completely obliterated by even low levels of collective effects. 
Above the threshold $\left(R_{\mathrm{th}}\right)$, the field in the cavity is a steady-state phenomenon and is thus available for study in some detail. Below threshold, the cavity field is dominated by cavity decay and is therefore a transient effect lasting for a cavity decay time after the interaction of the atom. This is the singleatom-effect region. As the evolution of the atom and cavity field is fully coherent, the atom and cavity field are entangled after their interaction. This entanglement is used, for example, to probe the cavity field in the normal operation of the maser. In the single-atom regime this can be exploited to create a transient field that exists in the cavity for a photon lifetime. As the atomic beam has a Poissonian distribution of arrival times, it can happen that, even in operation well below threshold, several atoms enter the cavity within a cavity decay time. Post-selective detection of such events has been used to observe the interaction of the field with up to three atoms under these conditions $[7,27-30]$.

\section{2 .3}

\section{Review of Experiments on Basic Properties of the One-atom Maser}

Atoms interacting with the cavity field undergo Rabi oscillations and their emission probability is determined by the accumulated Rabi phase when they leave the cavity. When the cavity field is initially in a coherent field state, the photon probability distribution is Poissonian, which results in dephasing of the Rabi oscillations causing their subsequent collapse. After collapse, the evolution of the atom and quantized cavity field results in a revival of the Rabi oscillations. These revivals occur periodically with a period determined by the square root of the average photon number. This phenomenon is known as "collapse-and-revival" and is a purely quantum mechanical feature with no classical counterpart [31].

Collapse-and-revival can also occur in the case of a thermal Bose-Einstein field, where the spread in the photon number is much larger than that of the coherent state. In this case, the pattern of oscillations is more complicated, and the pattern never collapses quite completely - but this is also an inherently quantum mechanical phenomenon since the classical counterpart to this field would cause the oscillation to decay to zero with no revival at all. Collapseand-revival in the presence of a thermal field was first observed by Rempe et al. in 1987 [32]. Elmfors et al. [33] subsequently performed a theoretical analysis of the experimental conditions and found excellent agreement between theory and experiment, thus clearly showing that the interpretation of the data as collapse-and-revival was accurate. In 1996, collapse-and-revival was also observed in the presence of a coherent field by Brune et al. [34] (see also Sect. 1.3.1). 
The coherent interaction of atoms with the cavity field produces non-classical states in the cavity. Fig. 1.2 shows that over a wide range of interaction times a relatively low $N_{\mathrm{ex}}$ is already sufficient to generate a non-classical maser field. This is the case because the vacuum field initiates transitions to the lower maser level. The sub-Poissonian statistics can be understood from Fig. 1.2, where the normalized average photon number $\langle n\rangle / N_{\text {ex }}$ is shown on the right as a function of the pump rate $N_{\mathrm{ex}}$. An increase in the photon number decreases the emission probability, driving the photon number to a smaller value. A decrease in the photon number, by contrast, increases the emission probability, driving the photon number up. This is a coherent interaction and it is therefore a quantum mechanical stabilization of the photon number, producing sub-Poissonian statistics. As the atoms are the source of the field, the field statistics can be related to the atomic statistics in a systematic manner [35]. The observation of sub-Poissonian atomic statistics was reported by Rempe, Schmidt-Kaler and Walther [36], and its relation to the sub-Poissonian photon distribution was first studied by Rempe and Walther [37] and later by Briegel et al. [35].

The reason for the sub-Poissonian atomic statistics is as follows: A changing flux of atoms changes the Rabi frequency via the stored photon number in the cavity. Adjusting the interaction time allows the Rabi nutation cycle to be chosen such that the probability of the atoms leaving the cavity in the upper maser level increases when the flux, and hence the photon number in the cavity, is raised. This leads to sub-Poissonian atomic statistics since the number of atoms in the lower state decreases with increasing flux and photon number in the cavity. This feedback mechanism can be neatly demonstrated through the anticorrelation of atoms leaving the cavity in the lower state. The fact that anticorrelation is observed indicates that the atoms in the lower state are more equally spaced than expected for a Poissonian distribution of the atoms in the beam. This means that when, for example, two atoms in the upper state enter the cavity close to each other, the second one performs a transition to the lower state with reduced probability.

The interaction with the cavity field thus leads to an atomic beam with atoms in the lower maser level showing number fluctuations which are up to $40 \%$ below those of a Poissonian distribution usually found in atomic beams. This is interesting because atoms in the lower level have emitted a photon to compensate for cavity losses inevitably present. Although this process is induced by dissipation giving rise to fluctuations, the atoms still obey sub-Poissonian statistics. As discussed above, this can also be seen as a consequence of the quantum-non-demolition situation achieved in the region $\Theta \approx 2 \pi$ and multiples thereof.

The field strongly fluctuates at positions where phase transitions occur, these being caused by the presence of two maxima in the photon number distri- 

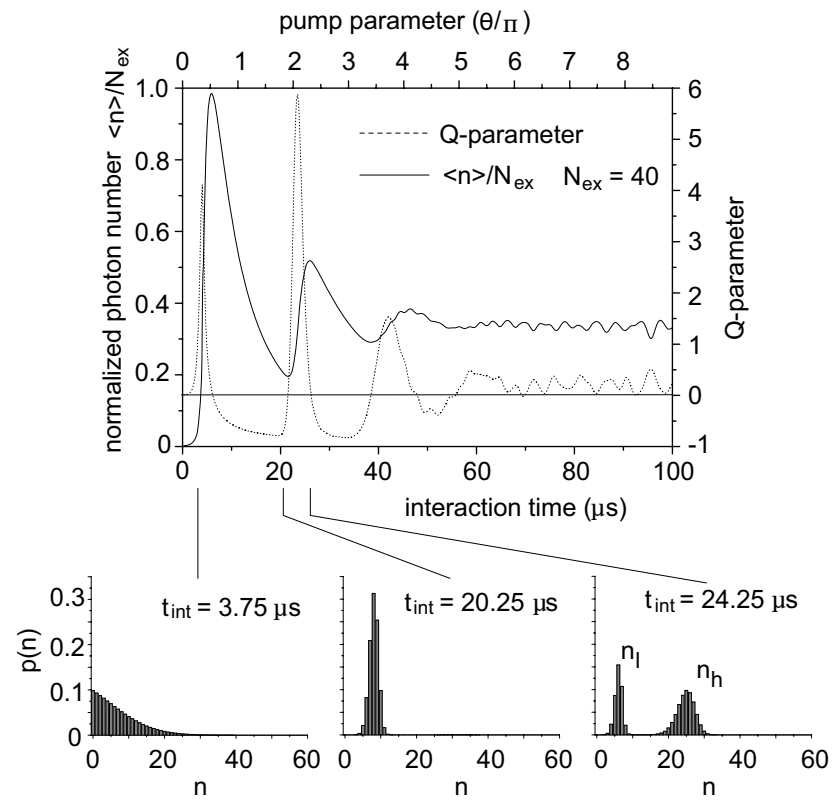

Fig. 1.3 One-atom maser or micromaser characteristics. The upper part of the figure shows the average photon number versus interaction time (solid curve) and the photon number fluctuations represented by the $Q$-factor (dotted line). In the lower part of the figure the steady-state photon number distribution $P(n)$ is shown for three values of $t_{\text {int }}$. The distribution on the left side corresponds to the maser threshold, that on

the right gives an example of the doublepeaked distribution associated with the quantum jump behavior. In this situation, the atom is back in the excited state and can again emit, leading to a higher steadystate photon number $n_{h}$. With increasing $t_{\text {int }}$ this part will grow and $n_{l}$ will decrease and disappear. A new jump occurs for pump parameters $\theta>3 \pi$.

bution $P(n)$ at photon numbers $n_{l}$ and $n_{h}\left(n_{l}<n_{h}\right)$; see Fig. 1.3. The phenomenon of two coexisting maxima in $P(n)$ was also studied in a semiheuristic Fokker-Planck (FP) approach [20]. There, the photon number distribution $P(n)$ is replaced by a probability function $P(v, \tau)$ with continuous variables $\tau=t / \tau_{\text {cav }}$ and $v=n / N_{e x}$, the latter replacing the photon number $n$. The steady-state solution obtained for $P(\nu, \tau), \tau \gg 1$, can be constructed by means of an effective potential $V(v)$ showing minima at positions where maxima of the probability function $P(v, \tau)$ are found. Close to $\Theta=2 \pi$ and multiples thereof, the effective potential $V(v)$ exhibits two equally attractive minima located at stable gain-loss equilibrium points of maser operation (see Fig. 1.3). The mechanism at the phase transitions is always the same: A minimum of $V(v)$ loses its global character when $\Theta$ is increased, and is replaced in this role by the next one. This reasoning is a variation of the Landau theory of firstorder phase transitions, with $\sqrt{v}$ being the order parameter. This analogy actually leads to the notion that, in the limit $N_{e x} \rightarrow \infty$, the change of the 


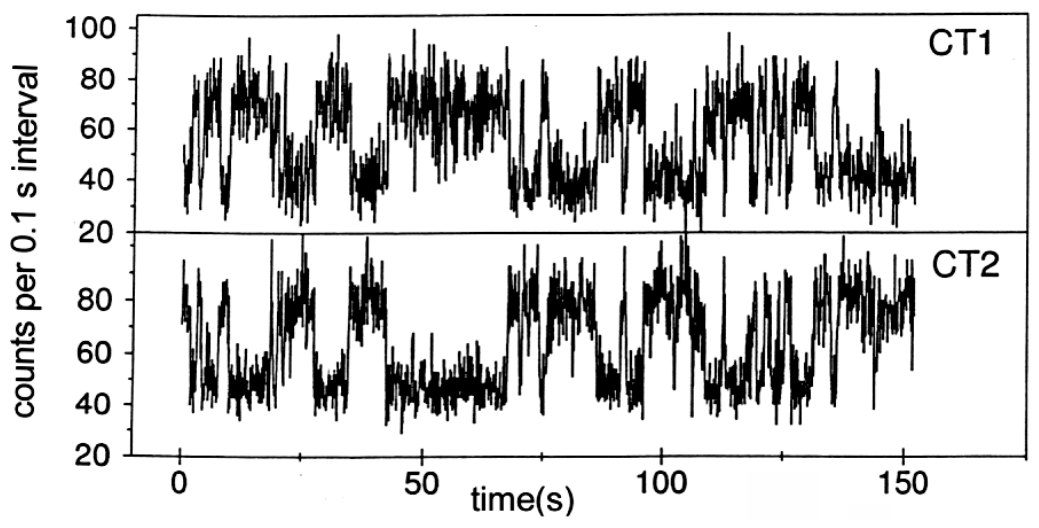

Fig. 1.4 Quantum jumps between two equally stable operation points of the one-atom-maser field. CT1 is the measurement of the upper state atoms, and CT2 that of the lower state atoms. It can be seen that both signals show counterphase behavior.

micromaser field around integer multiples $\Theta=2 \pi$ can be interpreted as firstorder phase transitions [38].

Close to first-order phase transitions long time constants of the field evolution are expected. This phenomenon was experimentally demonstrated, along with related phenomena such as spontaneous quantum jumps among equally attractive minima of $V(v)$, bistability, and hysteresis. Some of these phenomena are also predicted in the two-photon micromaser, for which qualitative evidence of first-order phase transitions and hysteresis has been reported. One example of this switching behavior will be discussed in the following. The maser was operated under steady-state conditions close to a first-order phase transition. The two maxima in $P(n)$ are manifested in spontaneous jumps of the maser field between the two maxima, with a time constant of $10 \mathrm{sec}$. This fact, and the relatively large pump rate, led to the clearly observable field jumps shown in Fig. 1.4. The two discrete values of the counting rates correspond to the metastable operating points of the maser, these being ca. 70 and ca. 140 photons: these two values correspond to two equally attractive minima in the FP potential $V(v)$. If one considers, for instance, the counting rate of lower-state atoms (CT2 in Fig. 1.4), the lower (higher) plateaus correspond to time intervals in the low (high)-field metastable operating point. If the actual photon number distribution is averaged over a time interval containing many spontaneous field jumps, the steady-state result $P(n)$ of the micromaser theory is recovered [38].

As noted above, between the regions of sub-Poissonian statistics, there are narrow regions of super-Poissonian statistics coinciding with thresholds in the photon number. These features are due to photon number phase transitions. 
In these regions, the steady-state photon distribution of equation (1.3) has two sub-Poissonian peaks that are well separated, so that the Fano-Mandel $Q$ parameter is positive (and relatively large). A photon number distribution of this particular kind gives rise to quantum jumps between these two subPoissonian peaks. This effect and the associated bistability that one would expect from this system have indeed been observed in the one-atom maser $[39,40]$.

\section{2 .4}

\section{Statistics of Detector Clicks}

In these experiments all information about the radiation field is second-hand, inasmuch as the experimenter can examine only atoms emerging from the resonator, but not the field itself. The Fock-state Rabi oscillations clearly show this. Although we do not measure the field itself, we are able to determine the photon distribution by analysis of an atom used as a probe of the field. Accordingly, in the steady-state maser dynamics, the atoms play a double role: (i) they pump the cavity and (ii) they provide the interaction diagnostics. The two roles interfere with one another because detection of the atom in a known final state leads to a quantum mechanical reduction of the photon state inside the resonator. Frequent detection is accompanied by quasi-permanent state reduction which can prevent the cavity field from relaxing to the steady state that would be reached if the atoms were left unobserved. Nevertheless, the steady-state properties determine the statistics of clicks recorded by the atom detectors. This method of calculation has been used rather successfully for determining the photon distribution in the cavity in recent experiments [30,41]. Furthermore, an extended analysis [42] predicts the probabilities of specific sequences of atoms (or, rather, of detector clicks) and therefore provides predictions of correlations between the internal state of the atoms that are successively detected in the field ionization zone. Measurement of these correlations therefore provides a method of assessing the accuracy of the formalism, and then other predictions of the model, which cannot be readily observed, such as the presence of entanglement, can be trusted as well.

The so-called "damping-bases method" [43] has been instrumental in extracting from the one-atom-maser theory predictions about atomic correlations and observed photon distributions [30,41,44]. These correlations have recently been observed and the correspondence between theory and experiment determined [44]. The success of this correspondence strongly suggests that other aspects of the theory, such as predictions of multiple atom entanglement, are also true. 
1.2 .5

Trapping States

Trapping states are a feature of steady-state operation of the one-atom maser, in which the field is peaked at a single photon number [13]. They occur in the one-atom maser as a direct consequence of the quantization of the cavity field. In fact, in the trapping states the quantum states of the radiation field become directly visible. The one-atom maser is the only system we know allowing to observe the quantum states of the radiation field directly. At low cavity temperatures the number of black-body photons in the cavity mode is reduced and trapping states begin to appear $[45,46]$. Under these conditions the sharp spikes appear that were not present in Fig. 1.2. These are the signature of the trapping states that occur at low temperatures because the emission probability $\mathcal{A}_{k}$ of equation (1.3) is then a dominant effect. The top plots in Figs. 1.5 and 1.6 show the effect of reduced temperature on the shape of the normalized photon number and the $Q$ parameter by comparing the curves expected for $100 \mathrm{mK}\left(n_{\mathrm{th}}=10^{-4}\right)$ and $500 \mathrm{mK}\left(n_{\mathrm{th}}=0.1\right)$. The two bottom plots show blown-up sections of the top plots to make the details clearer. In this case all of the curves are calculated for a cavity temperature of $100 \mathrm{mK}$, and we compare two pump rates, $N_{\mathrm{ex}}=20$ and $N_{\mathrm{ex}}=40$. With a change of pump rate, the trapping states remain fixed in position but their visibility changes dramatically. In the bottom plot of Fig. 1.6 the trapping states are manifested as regions of sub-Poissonian statistics with super-Poissonian regions on either side (this is seen more clearly in the curve for the lower pump rate).

Trapping states occur when $\mathcal{A}_{k}=0$, i.e. when, for zero thermal photons, emission of an atom is forbidden, so that the Fock space is effectively truncated and the photon number therefore naturally accumulates at this upper limit. Zero emission occurs when the atoms perform an integer number $(k)$ of Rabi cycles, i.e. when

$$
\sqrt{n_{k}+1} \Omega t_{\text {int }}=k \pi
$$

holds.

In fact, even for certain realistic steady-state operating conditions with a non-zero thermal photon number, the photon distribution can approximate a Fock state very well. Trapping states are characterized by the number of photons $n_{k}$ and the number of Rabi cycles $k$. The trapping state $\left(n_{k}, k\right)=(1,1)$ therefore refers to the one-photon, one-Rabi-oscillation trapped-field state. Equation 1.5 shows that a reliable indication of trapping states is that, unlike other features of the maser pump curve, they are independent of the pump rate, and thus a signature of trapping states is the observation of features in the maser pump curve, as a function of the interaction time, which will not move as a function of the pump rate. A second indication is the characteristic 


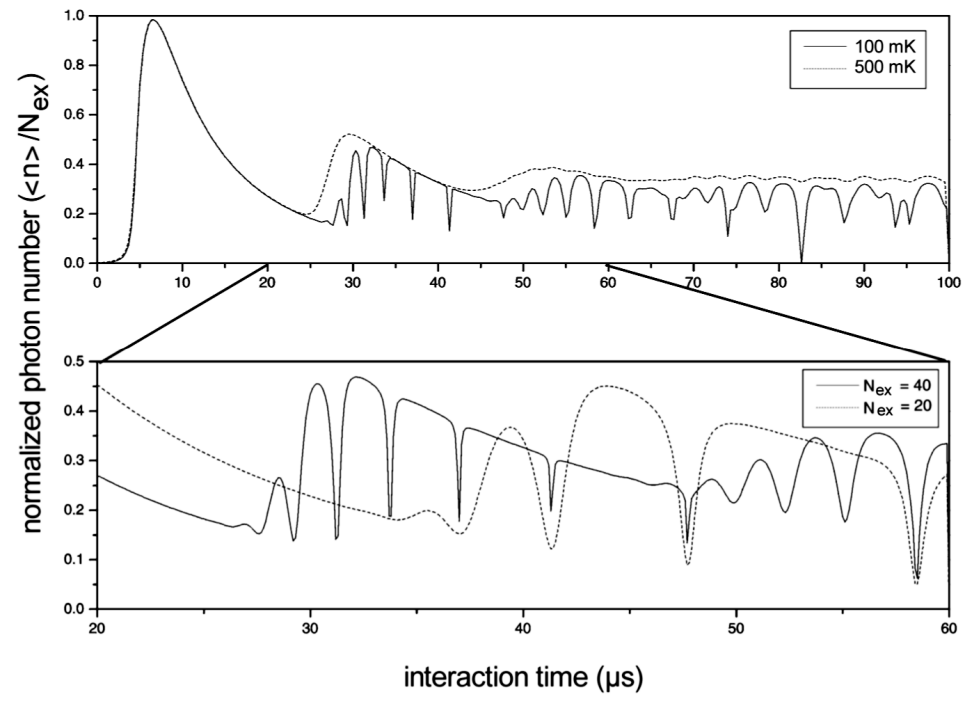

Fig. 1.5 One-atom maser at low temperature and high temperature. The top plot shows the effects of reduced temperature on the normalized photon number by comparing the expected curves for $100 \mathrm{mK}\left(n_{\mathrm{th}}=10^{-4}\right)$ and $500 \mathrm{mK}\left(n_{\mathrm{th}}=0.1\right)$. The bottom plot, which

shows a blown-up section of the top plot to make the details clearer, refers to $100 \mathrm{mK}$ and the curves compare two pump rates, $N_{\mathrm{ex}}=20$ and $N_{\mathrm{ex}}=40$. The trapping states remain fixed in position but their visibility changes dramatically.

oscillation of the statistical $Q$ parameter, as seen in the bottom plots of Figs. 1.5 and 1.6.

The effect of thermal photons (always present at finite temperatures) is to violate the trapping condition, so that the Fock space is no longer truncated. To observe trapping states, one therefore requires very low temperatures and highly stable conditions. Avoiding collective atomic effects and the effect of a finite atomic lifetime is also critically important for the realization of trapping states $[6,16-19,47]$. Trapping states are self-stabilizing against the decay of photons from the mode, since atoms are always available to replace photons lost to decay, thus restoring the trapping condition. With a careful choice of parameters, this self-stabilization can be exploited to produce photon number states in a wide range of operating conditions. This has recently been exploited for producing stable trapping state features [48] and also for creating photon number states on demand $[49,50]$.

The one-atom maser is the ideal system to realize trapping states. The cavity is operated at a temperature of $0.3 \mathrm{~K}$, leading to a thermal field of about $3 \times$ $10^{-2}$ photons per mode.

The first indication of trapping states in the maser field was reported in [46]. These results (corrected for the finite atomic lifetimes) are presented in Fig. 1.7 for two values of $N_{\mathrm{ex}}$ as a function of the interaction time. The cavity tem- 


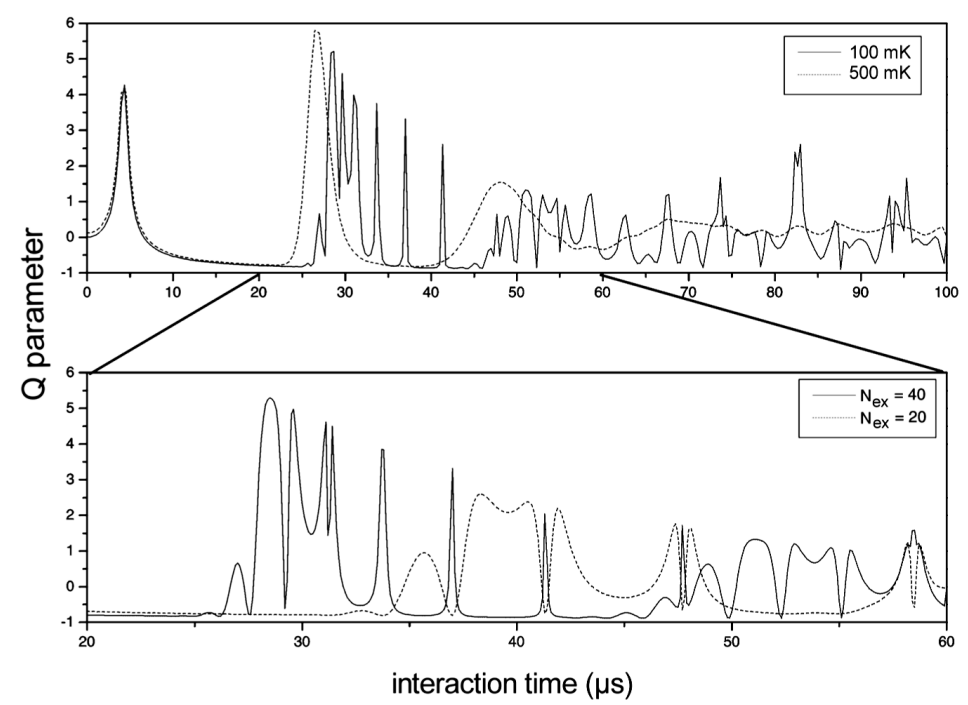

Fig. 1.6 One-atom maser at low temperature and high temperature. Same as Fig. 1.5, but here the $Q$ parameter is plotted. Note, in particular, that in the bottom plot the trapping states are manifested as regions of sub-Poissonian statistics with super-Poissonian regions on either side (this is seen more clearly in the lower-pump-rate curve).

perature was about $340 \mathrm{mK}$, which corresponds to a thermal photon number of 0.054 . This result shows good qualitative agreement with the Monte Carlo simulations by Raithel et al. [51]. The positions of the dips are preserved at increased pumping strength, as predicted. The reduction in visibility occurs for two reasons: Firstly due to narrowing of the peaks that occurs for increased pump rates (see Figs. 1.5 and 1.6) and secondly due to increased incidence of collective atom effects which act to reduce the visibility of the trapping state.

As the arrival times of the ground-state atoms were also collected in this experiment, it was possible to use equation 1.4 to extract the lower-state statistics of atoms leaving the cavity. The evaluation of this parameter from the experimental data is presented in the bottom plot of Fig. 1.7. For most of the positions where there is a trapping state sub-Poissonian statistics are also found. As the atom statistics and photon statistics are related, this is to be expected if the photon field is truly sub-Poissonian. One also finds a good qualitative relationship with the rapidly varying $Q$ parameter of the bottom plots in Figs. 1.5 and 1.6. Note that the vacuum is a coherent state and therefore the lower-state atom statistics should be Poissonian, as they are.

The trapping condition given in equation (1.5) is only accurate when the atoms are resonant with the cavity field. With detuning $\Delta \neq 0$, the vacuum 


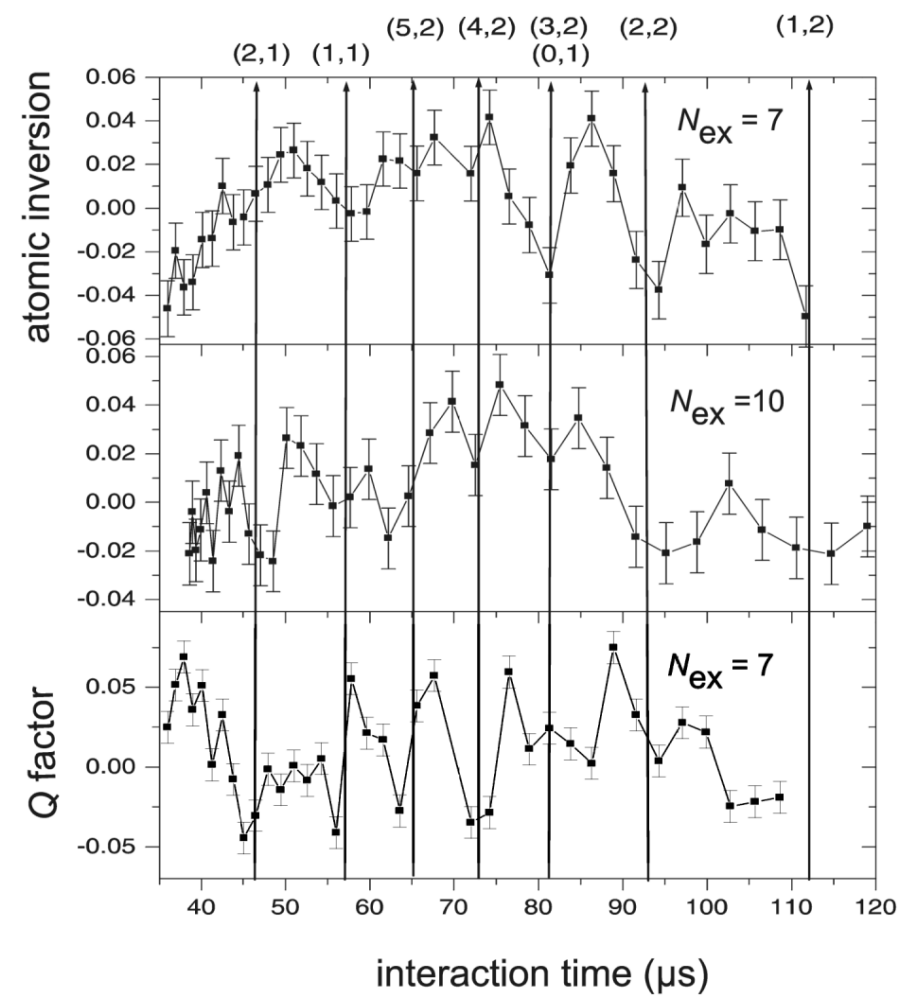

Fig. 1.7 Experimental measurement of trapping states in the maser field. The various trapping states are labeled by their $\left(n_{k}, k\right)$ pair of quantum numbers. Top and center: Trapping states appear as a reduced probability of finding a ground-state atom.

Bottom: Trapping states appear as regions

of sub-Poissonian statistics between regions of super-Poissonian statistics. An exception is the vacuum trapping state, which is the ground state of a coherent field, and therefore the atom counting should have Poissonian statistics. (Reproduced from [46].)

emission probability is given by

$$
P_{\mathrm{g}}\left(\Delta, t_{\text {int }}\right)=\frac{4 \Omega^{2}}{\Delta^{2}+\Omega^{2}} \sin ^{2}\left(\frac{1}{2} \sqrt{\Delta^{2}+4 \Omega^{2}} t_{\text {int }}\right) .
$$

This exhibits oscillatory behavior that results from Rabi flopping, but, since there is detuning, the observed flopping frequency is higher than that of the vacuum Rabi frequency. When detuning is used in an off-resonant situation, much more periods are thus observed than would occur when the experiment is performed on resonance.

In previous experiments, trapping states and thus the influence of Fock states, could be identified up to $n=5$ [46]. The set-up currently in use does not permit investigation of the purity of the Fock states obtained under the 
trapping condition. The dynamic generation of Fock states described in the next section, however, does allow the purity of Fock states to be measured.

\section{2 .6}

Trapping State Stabilization

Trapping states are self-stabilizing in that any photon loss due to cavity decay is replaced by a new photon from the pump atoms and, when the cavity field is in the trapping state, the field distribution is localized to a Fock state, where it will remain for a time determined by the rate of entry of thermal photons into the cavity. As shown above, thermal photons destroy the trapping condition. The best stability is thus achieved by adding a new loss mechanism that can remove any build-up of a field beyond the trapping state. This could be done by adding either lower state atoms to the beam (ground states in the trapping states) or a feedback mechanism that reduces the pump rate to compensate for increased emission [52].

However, a simpler and ultimately highly effective method is to operate the micromaser in cyclic operation, where the pump atoms are turned on and off for short periods (in comparison with the rate of the build-up of the thermal field) for pump and relaxation cycles. During the pumping cycle the cavity begins to fill with photons, approaching the corresponding $\mathrm{CW}$ condition; the pump rate is then turned off for a period to allow the field to relax a little before the next pumping cycle, thus correcting for the entry of thermal photons and allowing the field to resume the trapping condition in the next pulse. After many such cycles the field reaches a steady state that is a combination of the pumping cycle and the relaxation cycle. In this mode of operation, the entry of thermal photons only affects a single pulse, after which the cavity is allowed to relax to a photon number below the steady-state value. In such operation trapping states are a dominant feature of micromaser dynamics for a wide range of pump rates and thermal photon numbers.

The theoretical model for calculating the cyclically steady state of the maser (the steady state produced for a pulsed pump cycle) was developed to calculate photon distributions for the analytical analysis of Fock states and can be found in [41] and in a detailed review by Walther et al. [1]. See also [48, 49] and [50] for more details.

\subsection{7}

\section{Fock States on Demand}

In recent years there has been increasing interest in systems capable of generating photon fields containing a preset number of photons. This has chiefly arisen from applications requiring single photons, such as secure quantum communication and quantum cryptography. Fock states are also useful for 
generating multiple-atom entanglements in systems like the micromaser. In the micromaser the generated field and the pumping atoms are in an entangled state. This entanglement can be transferred by the field to subsequent atoms, leading to applications such as basic quantum logic gates.

A basic requirement for reliably preparing a field in a preset quantum state is the ability to choose the field state in a controllable manner [30]. Trapping states provide this means. Under trapping-state conditions, quantum feedback between the atoms and the field acts to control the cavity photon number. Trapping states can thus be used to provide photons on demand. Under ideal conditions, the micromaser field in a trapping state is a Fock state, but when the micromaser is operated in a continuous-wave mode, the field state is very fragile and highly sensitive to external influences and experimental parameters. However, in pulsed operation, as opposed to continuous-wave operation, trapping states are more stable and more practical, and can be used over a much broader parameter range than in continuous-wave operation [49]. An additional advantage is that the number of photons generated can be investigated by a probe atom after preparation.

To demonstrate the principle of Fock state preparation, Fig. 1.8 shows a simulation of a sequence of 20 pulses of the pumping atoms in which an average of seven excited atoms per pulse are present. Two operating conditions are present to compare conditions outside trapping conditions $\left(g t_{\text {int }}=1.67\right)$ with the $(1,1)$ trapping states $\left(g t_{\text {int }}=2.2\right)$. Below the pulse sequences, two distributions show the probability of finding $0-5$ atoms (and hence photons) per pulse in the cavity.

Under the trapping condition, only a single emission event occurs, which produces a single lower-state atom, leaving a single photon in the cavity. As during an atom pulse the atom-cavity system is in the trapping condition, the emission probability is reduced to zero after that and the photon number is stabilized. The variation of the time when an emission event occurs during the atom pulses in Fig. 1.8 is due to the Poissonian spacing of upper-state atoms entering the cavity and the stochasticity of the quantum process. The lower part of Fig. 1.8 shows the photon number distribution resulting from this process. In Fig. 1.8a the broader photon number distribution is due to the absence of a feedback stabilization.

\section{2 .8}

\section{Dynamical Preparation of $n$-photon States in a Cavity}

In this section we describe an alternative method of generating number states that also permits the analysis of the purity of the states generated to be unambiguously determined. We start the discussion of the method with some general remarks. 


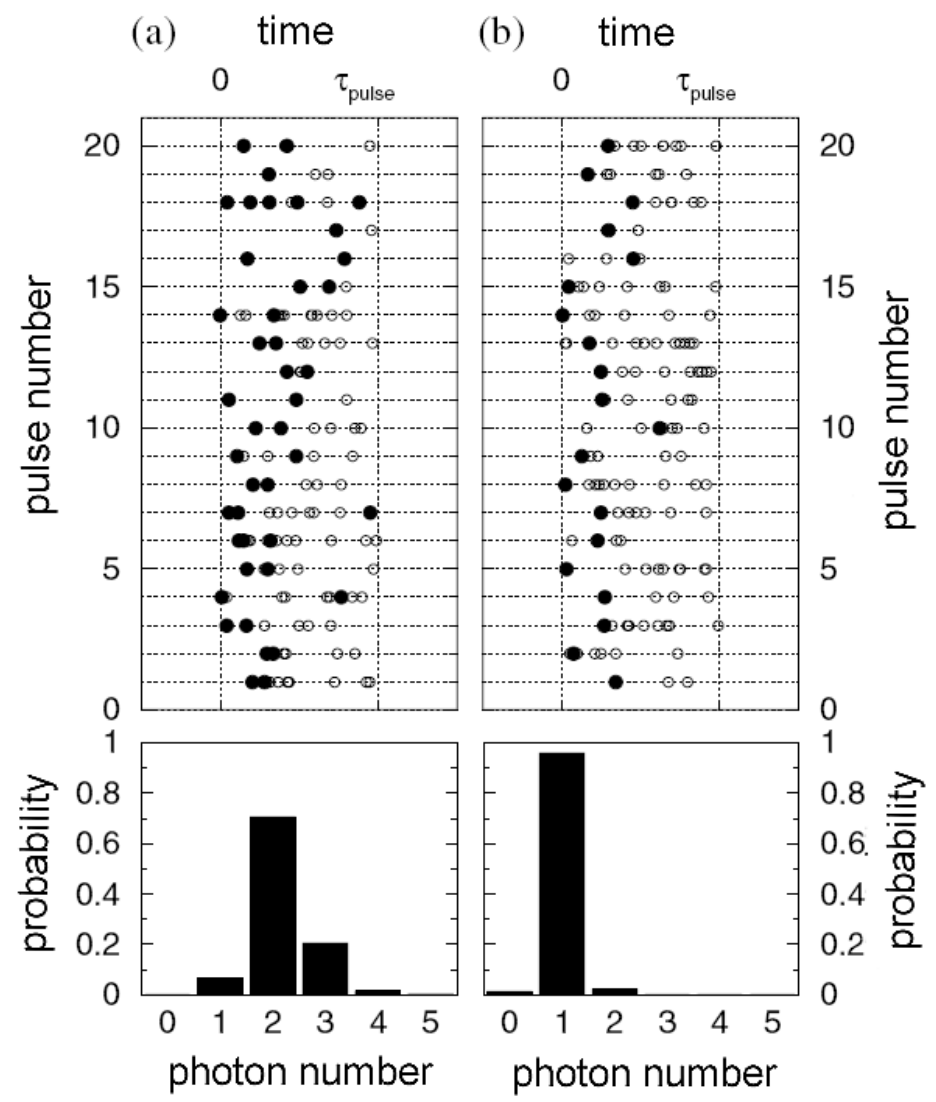

Fig. 1.8 A simulation of a subset of twenty successive pulses of the excitation laser and the associated probability distribution for photons or lower-state atom production (solid circles represent lower-state atoms and blank circles represent excited-state atoms). The start and finish of each pulse is indicated by the vertical dotted lines marked 0 and $\tau_{\text {pulse }}$,

respectively. The two operating conditions are (a) outside the trapping-state conditions $\left(g t_{\text {int }}=1.67\right)$, resulting in a broad photon number distribution, and (b) the $(1,1)$ trapping state $\left(g t_{\text {int }}=2.2\right)$ with a near-Fock-state distribution. Both distributions are subPoissonian but they are readily distinguishable experimentally. The figure is taken from [49].

When the atoms leave the cavity in a one-atom maser experiment, they are in an entangled state with the field. If the field is in an initial Fock state $|n\rangle$, then the interaction of an atom with the cavity leaves the cavity field in a superposition of the states $|n\rangle$ and $|n+1\rangle$ and the atom in a superposition of the internal atomic states $|e\rangle$ and $|g\rangle$. The entangled state can be described by

$$
|\Psi\rangle=\cos (\phi)|e\rangle|n\rangle-\mathrm{i} \sin (\phi)|g\rangle|n+1\rangle,
$$

where the phase parameter $\phi$ depends on the interaction time. The stateselective field ionization measurement of the internal atomic state reduces the field to one of the states $|n\rangle$ or $|n+1\rangle$. State reduction is independent of the 
interaction time, hence a ground-state atom always projects the field onto the $|n+1\rangle$ state independently of the time spent in the cavity. This results in an a priori probability of the maser field being in a specific but unknown number state [53]. If the initial state is the vacuum, $|0\rangle$, then a number state is created in the cavity that is equal to the number of ground state atoms that were collected within a suitably small fraction of the cavity decay time. This is the essence of the method of preparing Fock states by state reduction that was proposed by Krause et al. [53].

In a system like the micromaser there is no dissipative loss during the interaction and an atom in the cavity undergoes Rabi oscillations. That is, the relative populations of the excited and ground states of the atom oscillate at a frequency $\Omega \sqrt{n+1}$. As mentioned above, the atomic inversion has been experimentally investigated. In the presence of dissipation, a fixed photon number $n$ in a particular mode is not observed and the field always evolves into a mixture of such states. The inversion is therefore generally given by

$$
I\left(t_{\text {int }}\right)=-\sum_{n} P_{n} \cos \left(2 \Omega \sqrt{n+1} t_{\text {int }}\right),
$$

where $P_{n}$ is the probability of finding $n$ photons in the mode.

The method used in this experiment corresponds to a pump-probe experiment in which pump atoms prepare a quantum state in the cavity which is subsequently measured by a probing atom by studying the Rabi nutation. The signature that the quantum state of interest has been prepared is simply the detection of a definite number of ground-state atoms. To verify that the correct quantum state has been projected onto the cavity, a probe atom is sent into the cavity with a variable, but well-defined, interaction time in order to allow measurement of the Rabi nutation. As the formation of the quantum state is independent of the interaction time, we need not change the relative velocity of the pump and probe atoms, thus reducing the complexity of the experiment. In this sense, we are performing a reconstruction of a quantum state in the cavity using a method similar to that described by Bardoff et al. [54]. This experiment reveals the maximum amount of information on the cavity photon number that can be found.

The laser beam populating the upper state was pulsed to create short excitation pulses with only a small probability of exciting one atom; thus when two were observed (i.e. the pump and probe), it is highly probable that not more than two were excited. In fact, to create and detect an $n$ photon number state in the cavity, $n+1$ atoms are required. That is, $n$ atoms to create the number state and the final atom as a probe of the state. With $40 \%$ detector efficiency and the assumption that the probability of missing a count is statistically independent, there is an approx. $1 \%$ probability of the state preparation being incorrect because an atom escapes detection. Figs. 1.9(a-c) displays three Rabi 

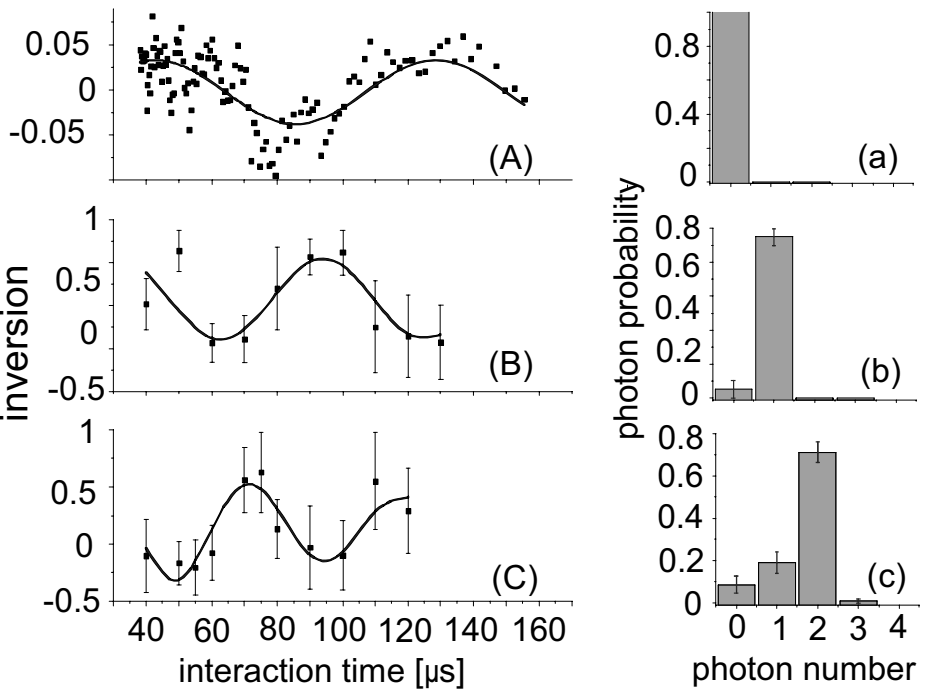

Fig. 1.9 Plots (A), (B) and (C) on the left present three Rabi oscillations for the number states $n=0,1$, and 2. Plots (a), (b) and (c) on the right plots display the coefficients $P_{n}$. The photon distribution $P_{n}$ was calculated for each Rabi cycle by fitting equation (1.7) to each plot for the set of photon numbers, $n=0$ to $n=3$. The relative phase of the Rabi oscillation was fixed since all the atoms enter in the excited state of the maser transition. In each fit, the highest probability was obtained for the target number state. Unlike the $n=1$ and $n=2$ Rabi cycles, the $n=0$ oscillation of plot $(\mathrm{A})$ was obtained in the steady-state operation

of the micromaser in a very low-flux regime. The fit to this curve was performed for Rabi cycles from $n=0$ to $n=2$. The low visibility of this curve was due to the low flux (one atom per second) which was required to reduce the steady-state operation of the micromaser to below-threshold behavior; hence detector dark counts become comparable to the real count rates and therefore contribute to a large background. During the Rabi cycle the cavity photon number changes periodically. At the maxima there is one photon more than at the minima. (Reproduced from [30].)

cycles obtained by measuring the inversion of a probe atom that followed the detection of $n=0,1$, and 2 ground-state atoms, respectively.

Because of the long waiting times for three atom events, the $n=2$ Rabi data were more difficult to collect than the other two measurements. The data collection time became substantially longer as the interaction time was increased, and then background effects have a higher impact on the data. The fit to the $n=2$ data includes an exponentially decreasing weight, so that measurements obtained for longer interaction times have less significance than those at short times.

The fact that pure number states were not obtained here is due to dissipation in the time interval between production and analysis of the cavity field. The simulations described in the following demonstrate that one could generate number states with a purity of $99 \%$ for the $n=1$ state and $95 \%$ for the $n=2$ 


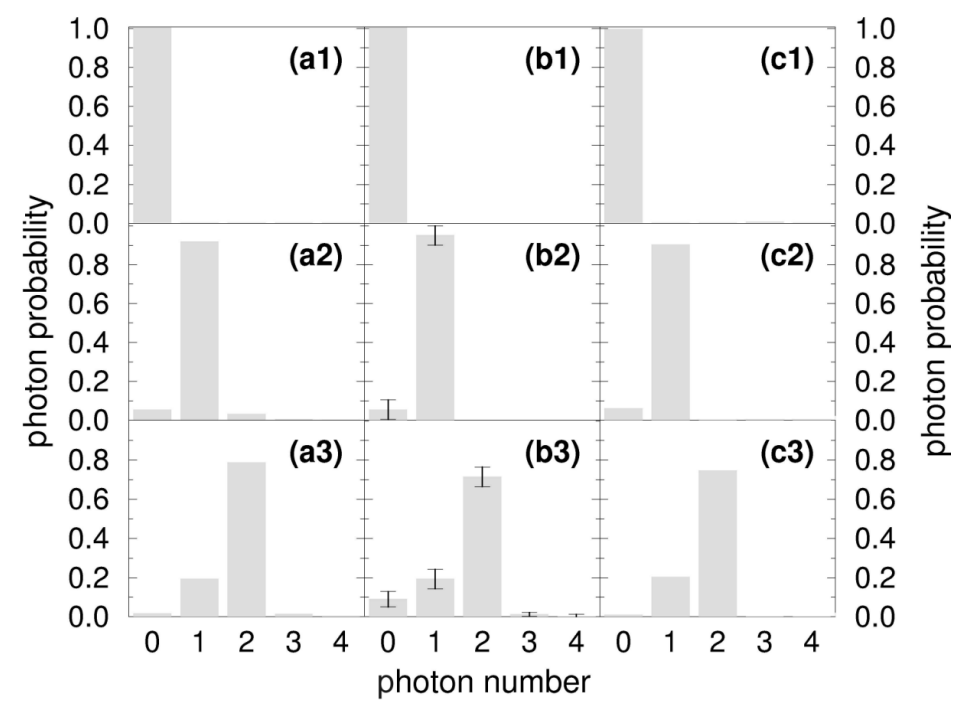

Fig. 1.10 Comparison between theory and experimental results on the purity of number states. The columns represent photon distributions obtained from: (a) a theoretical simulation of the current experiment; (b) the current experimental results; and (c) a theoretical model that extends the current experiment to the steady state at the positions

of the trapping states. The agreement between the two theoretical results and the experimental result is remarkable, indicating that dissipation is the most likely loss mechanism. Without dissipation, i.e. at the moment of generation the purity of the states is $99 \%$ for $n=1$ and $95 \%$ for $n=2$. (Reproduced from [30].)

state at the time of generation, which is then modified by dissipation between production and measurement.

For the simulations two idealizing assumptions were made: thermal photons are only taken into account for the long-term build-up of the cyclically steady state and Gaussian averaging over the velocity spread of the atoms is considered to be about 3\%. Included in the calculations are the exponential decays for the cavity field during the pulse when either one photon (for $n=1$ ) or two photons were deposited one by one (for $n=2$ ), changing the photon number distribution. The simulations also average over the Poissonian arrival times of the atoms. The details of the calculation are discussed in [41]. They are compared with the experimental results in Fig. 1.10(a) and (b).

As dissipation is the most essential loss mechanism, it is interesting to compare the purity of the number states generated by the current method with that expected for trapping states (cf. Fig. 1.10(c)). The agreement of the purity of the number states is striking. The trapping state photon distribution is generated in steady state, which means that whenever the loss of a photon occurs the next incoming atom will restore the old field with a high probability. The non-zero amplitudes of the states $|0\rangle$ in Fig. 1.10(c2) and $|1\rangle$ in Fig. 1.10(c3) are due to dissipative losses before restoration of a lost photon 


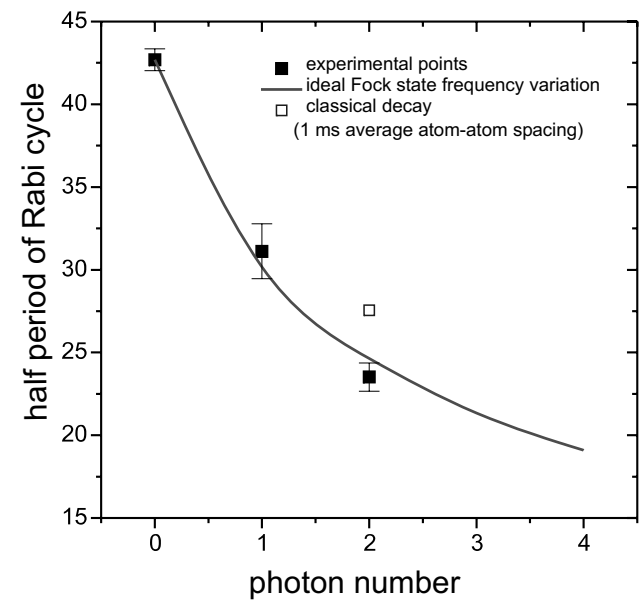

Fig. 1.11 Dependence of the Rabi frequency on the photon number. A single sine fit to each of the three Fock states is plotted versus the theoretical variation of frequency as a function of photon number. The coupling constant $36.8 \mathrm{krad} / \mathrm{s}^{-1}$ is the optimum fit to the data. The figure is taken from [48].

which is not replaced immediately but after a time interval dependent on the atom flux. The atom rate used in these calculations was 25 atoms per cavity decay time, or an average delay of $1 \mathrm{~ms}$. This can be compared to the delay between the preparation and probe atoms in the present experiment. In the steady-state simulation loss due to cavity decay determines the purity of the number state; in the limit of zero loss the state measurement is perfect. It can therefore be concluded that dissipative loss due to cavity decay in the delay to a probe atom largely determines the measured deviation from a pure number state. There is, of course, the question of the influence of the thermal field on the photon distribution. By virtue of the selection process in the current experiment we reduce the influence of the thermal field by only performing measurements of the field state following a trigger of ground-state atoms. Hence the state of the field is well known. The simulations for the steadystate case were therefore performed for a temperature of $100 \mathrm{mK}$, which makes the influence of the thermal field on the steady state correspondingly low. While this is not the first observation of Fock states in general (the $n=1$ Fock state having been observed earlier [55]), it is the most detailed study of the interaction of an atom with a quantized mode that has been carried out to date. Here we see the creation of a field state by state reduction and the observation of the interaction of this state with a probe atom.

The unambiguous generation of Fock states of the radiation field described in the previous section allows an interesting observation concerning the decay of the photons. Quantum fields decay discretely. This can be neatly observed 
by looking at the variation of the Rabi frequency in time observed after the Fock state generation. The results are shown in Fig. 1.11. The discrete decay follows the solid curve in the figure, whereas a classical and continuous decay would result in a larger period of the Rabi frequency given by the square at photon number state $\mathrm{n}=2$ [48].

\section{2 .9}

\section{The One-atom Maser Spectrum}

The properties of the micromaser we have discussed so far were all connected with the population of the maser levels or with the diagonal elements of the radiation-density matrix. In the following we would like to discuss a featurethe micromaser spectrum - which depends on the nondiagonal elements [56].

The micromaser spectrum is determined by the decay of the expectation value of the electric field [57] of the maser field

$$
\langle E(t)\rangle \sim \sum_{n=0}^{\infty}(n+1)^{1 / 2} \rho_{n, n+1}(t)
$$

In order to calculate the spectrum and the linewidth $D$ of the micromaser an analytical approach was used [56] whereby two novel features, quite dis-

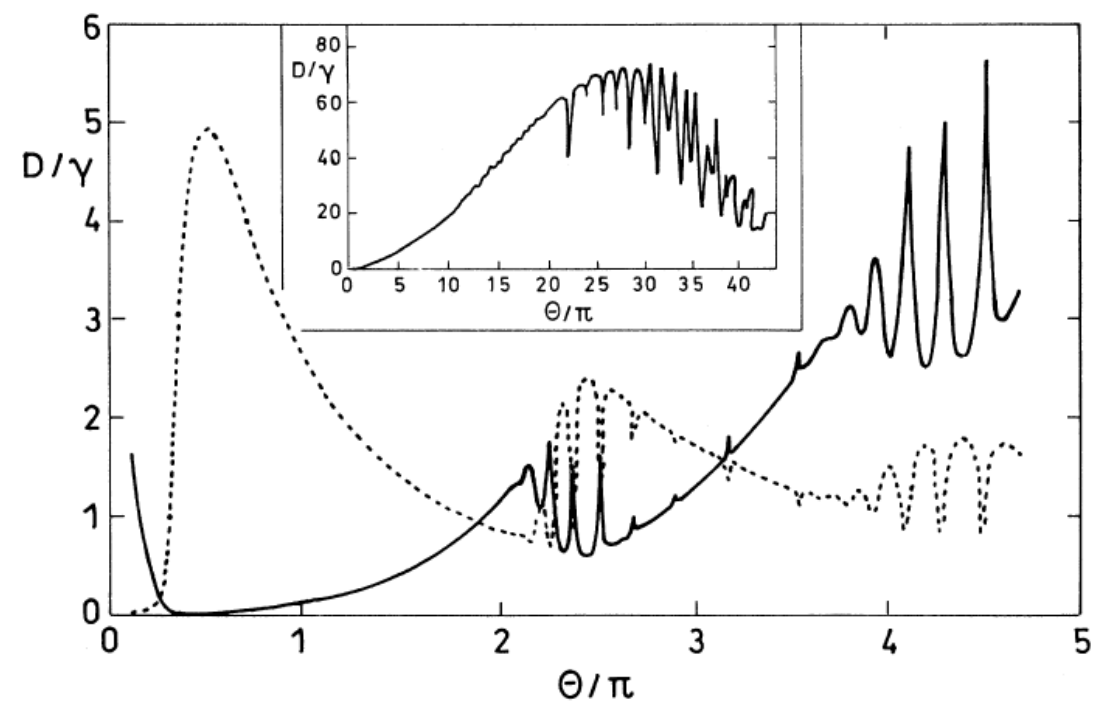

Fig. 1.12 The relative linewidth $D / \gamma$ based on equation 1.10 (solid curve and mean photon number $\langle n\rangle / 10$ (dotted curve) as a function of the pump parameter $\Theta=N^{1 / 2} g \tau$ for $N=50$ and mean thermal photon number $n_{b}=10^{-4}$. The inset sows the exact relative linewidth $D / \gamma$ based on a numerical solution of the density matrix for large pump parameter $\Theta$ for $N=20$ and $n_{\text {th }}=1$. 
tinct from the familiar Schawlow-Townes linewidth [57], show up. As can be seen in Fig. 1.12 (i) the trapping states impress sharp resonances onto $D$ as a function of the pump parameter $\Theta$. (ii) For large values of $\Theta$ the linewidth $D$ decreases, and can even oscillate, a phenomenon alien to the monotonic dependence of the Schawlow-Townes linewidth.

Fig. 1.12 is based on the equation for the linewidth [56]:

$$
D=4 r \sin ^{2}\left[\frac{g \tau}{4 \sqrt{\langle n\rangle}}\right]+\frac{\gamma\left(2 n_{t h}+1\right)}{4\langle n\rangle} .
$$

This equation is derived from the exact spectrum using a detailed balance condition and the fact that the equation of motion for the density matrix elements $\rho_{n, n}$ only couples to the nearest neighbors $\rho_{n, n+1}$ and $\rho_{n, n-1}$. For the exact numerical treatment of the relevant density-matrix equations see $[58,59]$

In Fig. 1.12 the detailed behavior of the phase diffusion constant $D$ is depicted (solid line) as a function of the pump parameter $\Theta=\sqrt{N} g \tau$ for $N=50$ and $n_{\text {th }}=10^{-4}$ thermal photons. The sharp resonances in the monotonic increase of $D$ are reminiscent of the trapping states. To bring out this similarity in the same figure, the average photon number as a function of $\Theta$ (dotted curve) is shown. We note that the phase diffusion is especially large when the maser is locked to a trapping state, that is, when $\langle n\rangle$ is caught in one of those sharp minima. The equation 1.10 for $D$ reveals this behavior in the limit of short interaction times or large photon numbers, that is when $g \tau / 4\langle n\rangle^{1 / 2} \ll 1$. If the sine function is expanded, the familiar Schawlow-Townes linewidth [57] is obtained

$$
D=\frac{\alpha+\gamma\left(2 n_{\mathrm{th}}+1\right)}{4\langle n\rangle}
$$

where $\alpha=\gamma\left(N^{1 / 2} g \tau\right)^{2}=\gamma \Theta^{2}$.

The complicated pattern of the micromaser linewidth results from the complicated dependence of $\langle n\rangle$ on the pump parameter indicated in Fig. 1.12 by the dotted curve which enters in the denominator. We emphasize that the maser linewidth for the micromaser goes beyond the standard SchawlowTownes linewidth. The sine function in the formula for $D$ suggests in the limit of large $\Theta$ values an oscillatory behavior of the linewidth. The exact numerical result shown in the inset of Fig. 1.12 confirms that.

The experimental investigation of the micromaser spectrum is of great fundamental interest. As the nondiagonal field elements are involved, their measurement is not so easy to perform with the present set-up as the holes in the cavity for the atomic beam may perturb the nondiagonal contributions. Therefore several methods have been proposed allowing to measure the phase diffusion despite those perturbing effects [60-62]. All those methods are based on a heterodyne scheme. The maser field is displaced by an injected field and 
then afterwards measured by a probe atom. In this way a reconstruction of the field is possible just by the determination of the atomic inversion.

\section{3}

\section{Other Microwave Cavity Experiments}

In the following, we briefly review the cavity QED work performed by the group of $\mathrm{S}$. Haroche and co-workers at ENS in Paris.

The cavity of the ENS group has an open geometry made of two spherical superconducting mirrors in a Fabry-Perot configuration marked by $\mathrm{C}$ in Fig. 1.13. The transitions investigated by this group are at $51.1 \mathrm{GHz}$, corresponding to a transition between circular Rydberg states with principal quantum numbers 51 and 50 . The circular states are characterized by maximum orbital and magnetic quantum numbers. The valence electron is a thin torus centered on the atom's core. They are produced in the CB unit using velocityselective laser excitation. The velocity-selected atoms cross the cavity perpendicularly to the axis; the interaction length is about $6 \mathrm{~mm}$ in diameter. The decay time of the cavity is typically $1 \mathrm{~ms}$ (corresponding to $Q=3$. $10^{8}$ ). The entire set-up is cooled to about $1 \mathrm{~K}$ to minimize thermal noise $[7,29,63]$. At the thermal equilibrium the cavity mode contains about 0.7 thermal photons on the average, originating from the thermal field leaks. In order to remove this field at the beginning of each sequence atoms prepared in the lower state are transmitted through the cavity to absorb the thermal photons. At the end of this cooling sequence the mean photon number is reduced to 0.1 per mode. Detection of the Rydberg atoms is performed as in the Munich set-up by field ionization which can be done state-selectively (Sect. D, Fig. 1.13). Experiments are also performed where superpositions of the states are investigated. The set-up has for this purpose two additional classical fields $R_{1}$ and $R_{2}$ coupled to the atoms via two spherical cavities to allow Ramsey-type two-field interaction ( [64]). The signal fringes can be investigated to study changes of the phase and amplitude of the interaction between atom and cavity [65].

With this set-up a variety of experiments on the atom-cavity interaction were performed. A survey is given in the following. For a detailed review see [66].

\subsection{1}

Collapse-and-revival of the Rabi Oscillations in an Injected Coherent Field

As discussed above, the quantum dynamics depends on the photon statistics of the interacting field. We have already described the measurement of the dynamics in a thermal field in the micromaser cavity [32] and in a number 


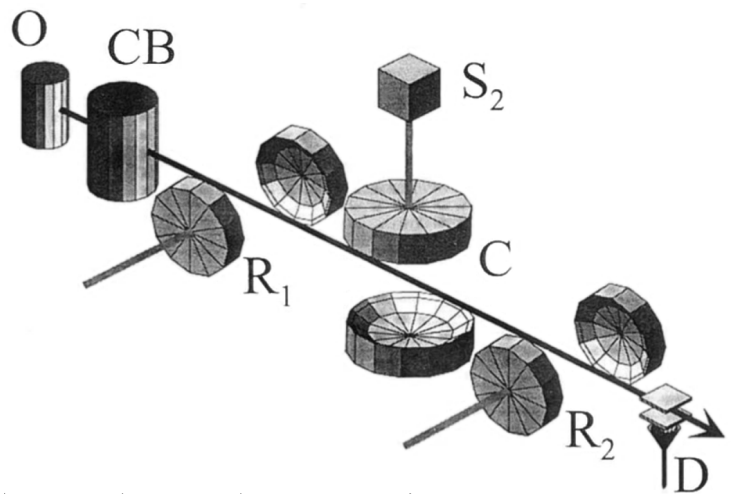

Fig. 1.13 Cavity-QED set-up used by the group of S. Haroche et al. at the Ecole Normale Superieure.

state field up to $\mathrm{n}=2$ [30]. Further experiments have been performed with the spherical cavity and an injected coherent field with an average photon number of $n=2$ [34]. The expected collapse-and-revivals [31] were observed.

Revivals of Rabi oscillations can also be induced by a time reversal pulse [67]. The technique was first proposed by Morigi et al. [68] and is suitable to distinguish contributions from coherent and incoherent processes in the evolution of the system. For this purpose the system is exposed to a short electromagnetic pulse after a free evolution during the time $T$ leading to a reversal of the system's unitary evolution. At time $2 T$ the system then resumes the initial state if no decoherence is present. This scheme was applied to investigate the atomic Rabi oscillations in a mesoscopic coherent field [67] with an average of 13 photons. The oscillation undergoes collapse due to the dispersion of the Rabi frequencies. This effect is unitary and reversible. After a time proportional to the field amplitude, the oscillation is expected to revive. When a $\pi$ phase shift is applied to the atomic coherence after the collapse time, an early induced Rabi revival is observed. The amplitude of this echo signal is sensitive to non-unitary decoherence processes.

\subsection{2}

\section{Atom-photon and Atom-atom Entanglement}

It has already been mentioned above that quantum Rabi oscillations provide a simple and flexible tool to entangle atoms and photons (see Sect. 1.2.2). If the Rabi oscillation in vacuum is interrupted mid-way ( $\pi / 2$ pulse) between the upper and lower atomic states, the final state of the atom-cavity field system is a maximally entangled state. In the event of the Rabi oscillation lasting twice as long ( $\pi$-pulse) the cavity and atom exchange their energy with maximum probability. An atom initially in the lower state of the transition when it 
enters the cavity containing one photon will end up with high probability in the upper state, leaving the cavity empty. This process can be used to transform an atom-field entanglement into an atom-atom one. When in the experiments two atoms cross the cavity one after the other, the first is initially in the upper level and the second in the lower one. The first atom undergoes a $\pi / 2$ Rabi pulse and gets entangled with the cavity. The second is subjected to a $\pi$ pulse, copying the cavity state and getting entangled in the process with the first atom. This entanglement was checked in various measurements on the final states of the two atoms and in studying their correlations [27,28]. See also [66] in connection with the generation of EPR pairs. For related work on entanglement with the micromaser see [44]. The generation of a GHZ state and EPR tests in the micromaser is described in [69] and [70].

\subsection{3}

\section{Atom-photon Phase Gate}

An atom-photon phase gate can be realized since after a full cycle of quantum Rabi oscillation ( $2 \pi$ Rabi pulse) the system reaches the initial state, but the phase of the wave function of the atom has undergone a $\pi$-shift. See also [71]. An atom initially in the lower state of the transition with one photon in the cavity therefore emerges in the same state, the photon being left in the cavity with high probability, but with the sign of the wavefunction of the atom being changed. If the cavity is initially empty, the sign of the atomic state is unaltered. When photon and atom are considered as quantum-bits carrying binary information, the $2 \pi$ Rabi pulse couples them according to the conditional dynamics of a quantum phase gate [63].

\subsection{4}

\section{Quantum Nondestructive-measurement of a Photon}

The principle of a quantum phase gate described above can also be used for nondestructive measurement of single photons in a cavity [7]. The phase change of the wavefunction of the atom occurring when exposed to a $2 \pi$ Rabi pulse can be transferred into an inversion of the phase of the fringe pattern of a Ramsey interferometer sandwiching the microwave cavity (Fig. 1.13). When the interferometer is adjusted to a fringe maximum, the photon number 0 or 1 is correlated to the final state of the atom. The method, however, does not allow one to measure deviations from a single photon state (see Sect. 1.2.6). See also [71].

Recently, it was also demonstrated that an experiment analogous to the photon detection described is also possible for nondestructive Rydberg atom counting using a mesoscopic field in a cavity [72]. The state-dependent atomic index of refraction being proportional to the atomic number shifts the classical 
field phase, which is changed into intensity information by a phase-sensitive homodyne procedure. This method may open the possibility of using Rydberg atoms for more applications in quantum information processing.

\section{3 .5}

\section{Wigner-function of a One-photon State}

Rydberg atoms are sensitive probes of a microwave cavity field that make it possible to determine its quantum state through determination of quasiprobability distributions which contain the information on the field density distribution. Furthermore, they exhibit the quantum properties of the field if present. The Husimi $Q$ and Wigner functions have been measured for atom cavity systems [73]. For direct determination of the Wigner function of a onephoton Fock state, by a method proposed in [71], see also Bertet et al. [74]. Lougovski et al. [75] showed that the Fresnel transform of measured Rabi oscillations provides an especially simple means of determining the Wigner function, being a much faster method than depending on the dispersive interaction between atoms and field. For the reconstruction of the Wigner function in the one-atom maser see [60] and [61].

\subsection{6}

\section{Multiparticle Entanglement}

When quantum Rabi pulses of variable duration and auxiliary Ramsey pulses are applied to successive atoms crossing a set-up of the kind displayed in Fig. 1.13, one can generate and analyze entangled states involving more than two particles. Such a procedure was demonstrated for the entanglement of up to three atoms [29]. The disadvantage of the experiment is that the atomic coherences cannot exit cavity C, which was modified in relation to Fig. 1.13 by an additional ring structure to increase the photon lifetime. A further limitation is that circular atoms are prepared with Poissonian statistics with a low mean value, requiring long data acquisition times. For a review on the multiparticle entanglement in the micromaser see [44]

\subsection{7}

\section{Schrödinger Cats and Decoherence}

For this experiment a small coherent field containing an average of 3 to 10 photons is prepared in cavity C (Fig. 1.13) by coupling it to an external coherent source. This field interacts with a single atom in a state superposition (produced by a $\pi / 2$ pulse in cavity R). The atomic transition and the cavity frequency are slightly off resonance so that the atom and the field cannot exchange energy but undergo dispersive frequency shifts. The atom-field 
coupling during the interaction time produces atomic-level-dependent dephasing of the field and generates an entangled state with the phase $\phi=g^{2} t / \delta$ ( $\mathrm{t}$ : interaction time, $\delta$ : detuning). After leaving field $\mathrm{C}$ the atom undergoes another $\pi / 2$ pulse in cavity $R_{2}$ identical to $R$ (Fig. 1.13). The signal in the field ionization detector shows Ramsey fringes [64] since the transition monitored can occur either in field $R_{1}$ or $R_{2}$ and these two "paths" are indistinguishable. The fringe contrast depends on $\phi$ and decreases with increasing $\phi$ due to decoherence since the "distance" between the interfering states varies as $D=2 \sqrt{n} \sin \phi$. The result obtained for the decoherence time is shorter than the photon decay time in the cavity [76]. The result agrees with simple decoherence theories. The open cavity $\mathrm{C}$ leads to a rather strong coupling to the environment, and so investigations with a better closed cavity would be desirable and this is being pursued by the ENS group.

\section{4}

\section{Cavity QED Experiments in the Visible Spectral Region}

The QED experiments in the optical region aimed at achieving strong coupling between atom and cavity field started in the early 1990s with experiments involving atomic beam transits through the mode of a high-finesse cavity $[77,78]$. The flux of atoms was small enough to make the average intracavity number roughly one. The one-atom vacuum Rabi splitting for the Cs transition $\left(6 S_{1 / 2}, F=4 \rightarrow 6 P_{3 / 2}, F^{\prime}=5^{\prime}\right)$ was observed. Similar observations were made by Feld et al. using Ba atoms [79]. The brief interaction time was a limitation in these experiments, and so subsequent experiments with clouds of cold atoms cooled to sub-Doppler temperatures were prepared in a magneto-optical trap (MOT) located a few millimeters above the cavity and released into the cavity. It was possible to observe in real time their individual trajectories and the duration of their transits [80]. This technique was used to extract the vacuum Rabi spectrum from the transmission of a weak probe.

\subsection{1}

\section{The One-atom Laser}

The one-atom laser is the optical analog of the one-atom maser. It has been theoretically discussed in many papers [81-90]. The gain medium is a single atom which couples photons into the resonant mode of the optical cavity. As expected, it is very different from a conventional and semi-classical laser and shows a variety of quantum properties.

The first laser oscillation with a single atom was observed by An et al. [91]. In this experiment a beam of Ba atoms transverses a single mode cavity with a finesse of $8 \cdot 10^{5}$. The atoms are excited by a $\pi$-pulse from the ${ }^{1} S_{0}$ ground 
state to the ${ }^{3} P_{1}$ excited state before they enter the cavity. The mean number of photons inside the cavity was 0.14 to 11 .

In a recent experiment $[5,92]$ a Raman-type transition was investigated where a pump beam from the $\left(6 S_{1 / 2}, F=3\right)$ ground state of the Cs atom to the $\left(6 P_{3 / 2}, F^{\prime}-3^{\prime}\right)$ excited state is applied perpendicularly to the cavity. The atomic population is transferred to $\left(6 S_{1 / 2}, F=4\right)$ via strong coupling to the cavity emitting a single excitation in the laser mode. A repumping beam is necessary to bring the population in the $6 S_{1 / 2} F=4$ state back to the ground state. With the set-up thresholdless lasing is observed. Furthermore, the oneatom laser exhibits photon antibunching and sub-Poissonian photon statistics. For further work on the one-atom laser see Sect. 1.4.4

1.4.2

\section{Atoms Pushed by a Few Photons}

The attractive potential in which the dressed states move, suggests that one could trap neutral atoms in potential wells of this kind, thereby exploiting the forces of just a few photons. This has indeed been suggested in [93]. The gravitational pull just mentioned is, however, much too strong. It cannot be compensated for by the forces that a few microwave photons could provide, and so one would need the microgravity environment of a satellite experiment to demonstrate the reality of these forces.

Circumstances are much more favorable in the optical domain. The light field of a small high-finesse optical resonator can catch a slowly moving atom. This was recently demonstrated in two experiments, one performed with cesium atoms at Caltech [94], the other with rubidium atoms at Garching [95].

In both experiments, single atoms were trapped by one or a few photons and, very impressively, the motion of the atom was tracked by analyzing the light transmitted through the resonator.

The actual atom-light interaction in these experiments needs to take into account the fact that the atoms may scatter the photons out of the resonator.

This is a rapidly developing and intense field of research, both experimentally and theoretically, and we refer the interested reader to the pertinent publications of recent years [94-103]. Let us just note that, in addition to the passive observation, active feedback has been successfully implemented [103], allowing the experimenters to prolong the storage time of the atom substantially.

While atomic confinement with quantized QED field offers an important advance in obtaining well-localized, trapped atoms, it remains preferable to decouple the trap from the QED interaction. A number of groups [104-107] have therefore realized optical dipole force traps, also known as far off-resonant traps or FORTs. They use a far-detuned optical beam inducing a dissipative, attractive force on an atom and since they are operated far off-resonance, they 

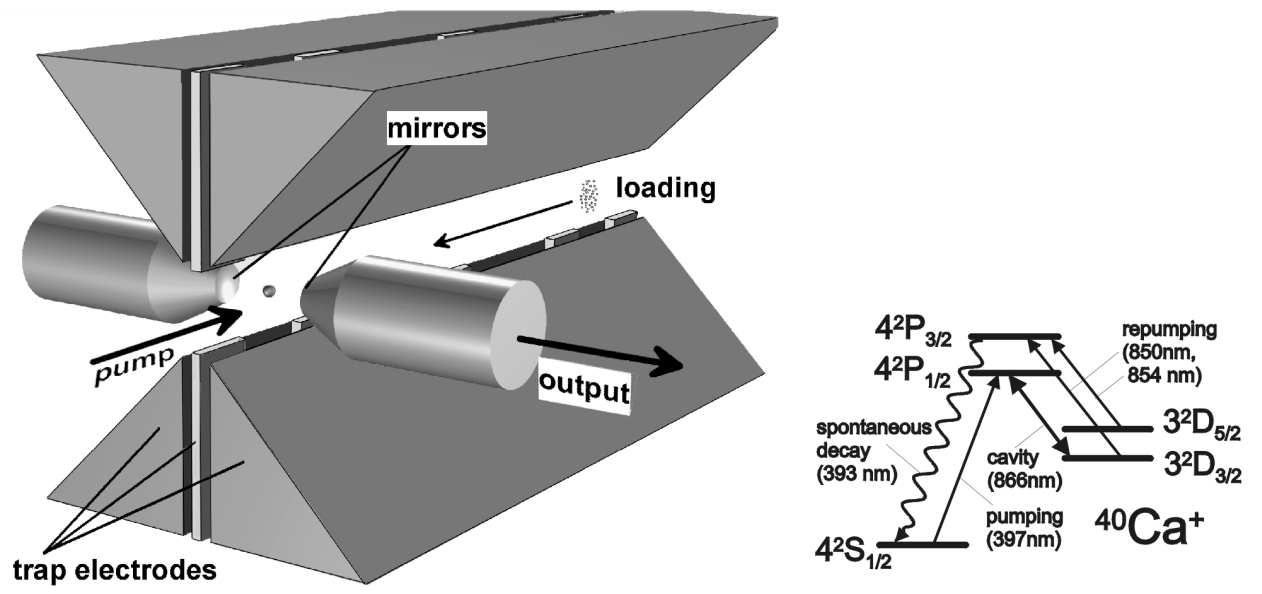

Fig. 1.14 Left: Set-up of the ${ }^{40} \mathrm{Ca}^{+}$ion trap laser at Garching. The loading trap at the rear is used to catch ions and cool them. A single ion is then transferred to the front trap between the mirrors of the optical cavity, where the ion is pumped and emits into the resonant resonator mode. (Reproduced

from [140].) Right: Level scheme. The lasing transition is $4^{2} \mathrm{P}_{1 / 2} \rightarrow 3^{2} \mathrm{D}_{3 / 2}$; the major pump transition is $4^{2} \mathrm{~S}_{1 / 2} \rightarrow 4^{2} \mathrm{P}_{1 / 2}$; the (re-)pumping from levels $3^{2} \mathrm{D}_{3 / 2}$ and $3^{2} \mathrm{D}_{5 / 2}$ to $4^{2} \mathrm{P}_{3 / 2}$ prevents the accumulation of population in dead ends; spontaneous decay from $4^{2} \mathrm{P}_{3 / 2}$ to the $4^{2} \mathrm{~S}_{1 / 2}$ ground state closes the cycle.

cause practically no atomic excitation. Examples where FORT traps were used in QED experiments are $[104,106,108]$. Using these arrangements allows one to keep an atom inside the cavity for a longer period, as was done in the experiments on single-photon generation [109] or on the one-atom laser [110]. Some considerable progress was recently achieved concerning the trapping time of an atom in a cavity [111]. In this paper it was shown that an orthogonal arrangement of cooling laser, trapping laser and cavity vacuum field gives rise to a combination of friction forces that act along all the three directions so that a single atom can be cooled in a high-finesse cavity, resulting in a trapping time of up to $17 \mathrm{~s}$. In a subsequent paper [112] using the same setup atoms were repetitively moved out and back into the cavity mode with a repositioning precision of $135 \mathrm{~nm}$. This makes it possible either to selectively address one atom or a string of atoms by the cavity field or to simultaneously couple two precisely separated atoms to a higher mode of a cavity.

Raman transitions have also been used to cool atoms in a cavity. This method was used to investigate the vacuum Rabi spectrum for one-and-the same atom, as opposed to other experiments using a FORT $[78,113,114]$ requiring averaging over a large ensemble of atoms. In the experiment investigating the Rabi splitting a single atom loaded into the FORT is irradiated with a probe beam whose frequency is varied over a range near the atom-cavity resonance, and the transmission of the probe beam is measured. This measurement cycle has 


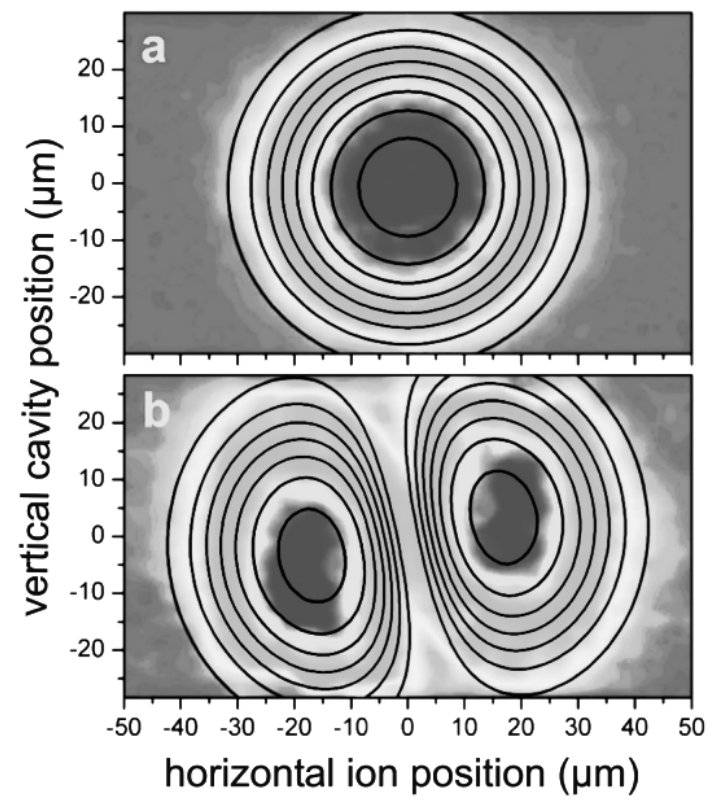

Fig. 1.15 Two-dimensional images of the cavity field taken over an area of $100 \times 60 \mu \mathrm{m}^{2}$. a) $\mathrm{TEM}_{00}$ mode. b) TEM 01 mode. In the horizontal direction the ion was moved, while vertically the cavity position was changed relatively to the ion. False colors represent the measured fluorescence count rate; the contour lines indicate the theoretical fluorescence pattern. Cf. the coloured plates in the Appendix.

to be interated with Raman cooling periods. The result agrees very well with the solution of the steady-state master equation of the system [115].

\section{4 .3}

\section{Single-photon Sources}

The controlled production of single photons is of fundamental and practical interest; they represent the lowest excited quantum states of the radiation field and have applications in quantum cryptography [116] and quantum information processing [117]. Common approaches use the fluorescence of single ions [118], single molecules $[119,120]$, color centers $[121,122]$ and semiconductor quantum dots [123-127]. However, the lack of control over such irreversible emission processes precludes the use of these sources in applications (such as quantum networks [128]) that require coherent exchange of quantum states between atoms and photons. The necessary control may be achieved in principle in cavity quantum electrodynamics. In these systems the directed emission can be controlled through the modes of the cavity. 
Sources of this kind have been proposed by different authors, e.g. [129-132]. All those schemes are based on a technique known as STIRAP (stimulated Raman scattering involving adiabatic passage) $[133,134]$ or variants thereof. The first observation of stimulated Raman scattering from a $\Lambda$-type three-level atom where the vacuum field of a high-finesse optical cavity is involved was realized by Hennrich et al. [99]. This scheme produces from a single atom one intracavity photon by means of an adiabatic passage sequence consisting of pump laser and cavity field. This photon is transmitted through a less reflecting mirror of the cavity. The transitions used were between hyperfine levels of the ${ }^{85} \mathrm{Rb}$ atom. The atoms used were pre-cooled in a MOT and released into the cavity. The experiment produced at most one photon per passing atom, but did not operate as a single photon source, since the continuous driving scheme simply mapped the random (Poissonian) atom statistics to the photons. This was later improved in a further experiment where pulsed driving was used together with pulsed recycling to allow a stream of several single-photon pulses to be produced on demand from one and the same atom, triggered by the detection of a first photon emitted from the cavity [135].

\subsection{4}

\section{Single-atom Laser Using an Ion Trap}

The ultimate limit of ultra-slowly moving atoms are atoms at rest. A single atom that stays put inside a high- $Q$ resonator will interact with the cavity mode for a long time and, provided the atom is suitably driven, may play the role of the active medium of a laser. This is the situation of the one-atom laser. Theoretical studies $[87,136,137]$ eventually confirmed that it is indeed possible to get laser operation with a single atom, as discussed in Sect. 1.4.1. Actual realization would require appropriate mechanical forces to keep the atom in place, and such forces are not easily provided.

A much more practical variant of this idea is therefore the ion-trap laser [88], where the active medium is a single ion trapped in a standard ion trap around which one positions the mirrors of the optical resonator; see Fig. 1.14. Careful studies $[89,90,138,139]$ demonstrate that the ${ }^{40} \mathrm{Ca}^{+}$ion is well suited to this purpose. One finds, for example, that a mean number of 5-10 photons builds up in the resonator (which corresponds to about $5 \times 10^{5}$ photons coupled out per second) and that, in some parameter ranges, the photon statistics may be sub-Poissonian. Experiments for actual realization of an ion-trap laser of this kind are well under way at Garching.

The strength of the ion-photon coupling depends on the location of the ion relative to the nodes and anti-nodes of the mode function in question. It is therefore mandatory to have good control of the ion's position. In fact, the experimenters are able to push the ion around at will (or, equivalently, to 
change the mirror positions), as is demonstrated by the measurement reported in Fig. 1.15. This plot shows a two-dimensional map of the mode function obtained by recording the fluorescence light while scanning the ion's position across a plane perpendicular to the resonator axis. The trapped ion can thus serve as a high-resolution probe of the optical field inside the cavity [140]. Owing to the rest motion of the trapped ion a resolution of the order of 40 $\mathrm{nm}$ has been achieved. The permanent and fully controllable coupling of a particle to the field with a well-defined strength is a unique property of ion-trap cavity quantum electrodynamics and provides the basis for the ioncavity experiments described here. In the following, we would like to discuss a single photon source on the basis of a trapped single ion.

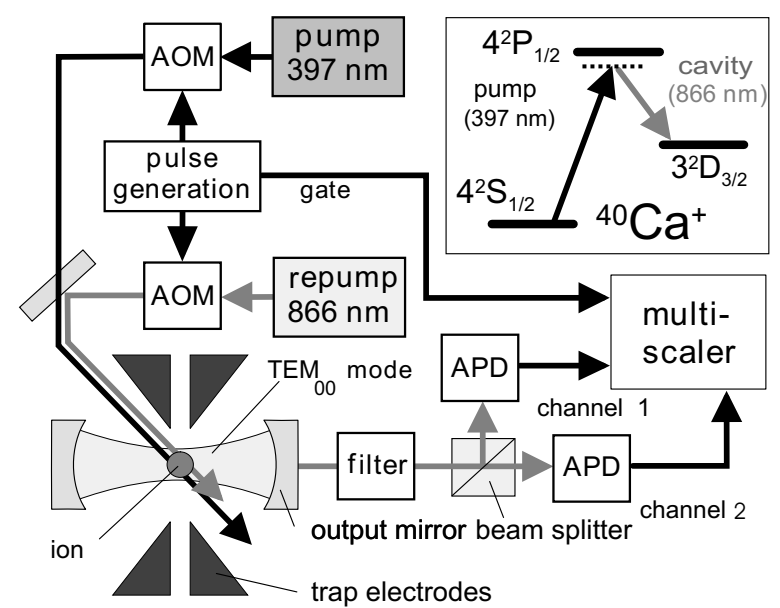

Fig. 1.16 Experimental set-up outlining the excitation and detection scheme for single photons. Not shown is an additional laser at $894 \mathrm{~nm}$ which is resonant with a cavity mode not coupled to the ion and which is used for stabilizing the cavity length. In the path to the detectors, this locking beam is suppressed by a series of filters, providing

an attenuation of $10^{11}$ at $894 \mathrm{~nm}$. The laser intensities are controlled with acousto-optic modulators (AOMs). The inset shows the relevant levels of the ${ }^{40} \mathrm{Ca}^{+}$ion used for single-photon production. The pump laser and cavity are red-detuned with respect to the $P_{1 / 2}$ level.

The first step of the protocol for generating a single-photon pulse is to apply radiation at 397 and $866 \mathrm{~nm}$ for $3 \mu$ s from the side of the cavity to laser-cool the ion and thus ensure its proper localization. Subsequently, the initial electronic state $S_{1 / 2}$ is prepared by optical pumping at $866 \mathrm{~nm}$ for $0.5 \mu \mathrm{s}$. A single photon is produced by driving a cavity-assisted Raman transition to the $D_{3 / 2}$ level by means of a pump pulse at $397 \mathrm{~nm}$ with a predefined intensity profile of up to 6 $\mu$ s duration. The sequence is repeated at a rate of $100 \mathrm{kHz}$. The photon pulse is emitted from the cavity through one mirror with a transmissivity of 600 p.p.m., 100 times larger than that of the opposite mirror. The Gaussian mode emanating from this mirror is focused on an avalanche photodiode (APD) 

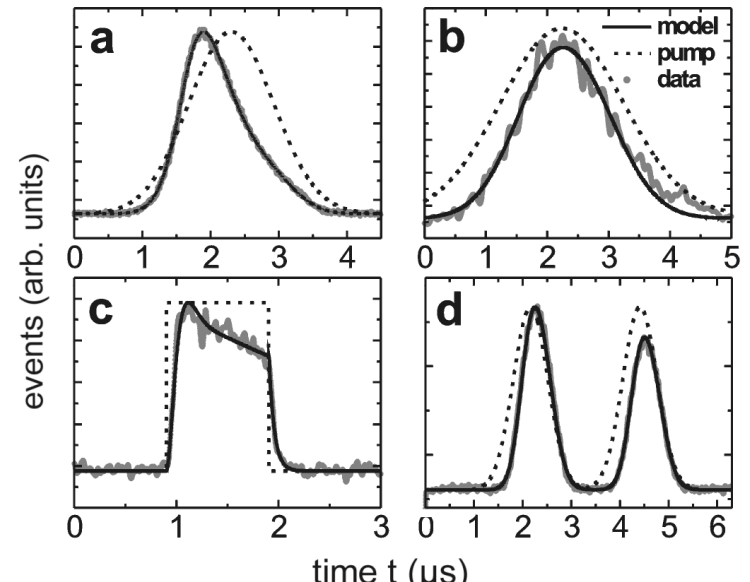

Fig. 1.17 Single-photon pulse shapes (gray the optical fields. Plots a) and d), obtained line) for different pump laser profiles, indicated by the dotted line (not to scale). a) Strong Gaussian pump. b) Weak Gaussian pump. c) Square-wave pump. d) Double-peaked pump. The superimposed black curves show the results of a density-matrix calculation, taking into account the full Zeeman structure of the ${ }^{40} \mathrm{Ca}^{+}$levels, as well as the polarization of with the best statistics, demonstrate the nearly perfect agreement between the model and the experimental data, confirming that the waveform of a single photon can be deterministically controlled. In Figs. a) and d) the experimental and theoretical curves are practically identical so that they do not show up as separate curves.

after passing a series of four optical filters and a spatial filter. In this way, light from an auxiliary laser used for locking the cavity length and from other sources of stray light is reduced to a level well below the dark-count rate of the detectors used.

With the position of the ion and hence the ion-field coupling fixed, the distribution of photon detection times exactly follows the shape of the singlephoton pulse. By tailoring the intensity profile of the driving pulse, we can imprint an arbitrary temporal structure on the waveform of the photon, which is then precisely reproduced in every emission event. The single-photon pulse shape is extracted from the time records by accumulating the probability distribution of photon arrival times relative to the pump-pulse trigger. Experimental data obtained for intense Gaussian pumping based on the evaluation of over 400,000 photons continuously generated from a single ion are presented in Fig. 1.17a. Because the photon pulses are identical, the time distributions obtained constitute a measurement of the waveform of a single photon. This is confirmed by comparing the data with calculated singlephoton pulse shapes, based on coherent Raman coupling of a calcium ion to a single cavity mode [141]. 
delay time $\tau(\min )$

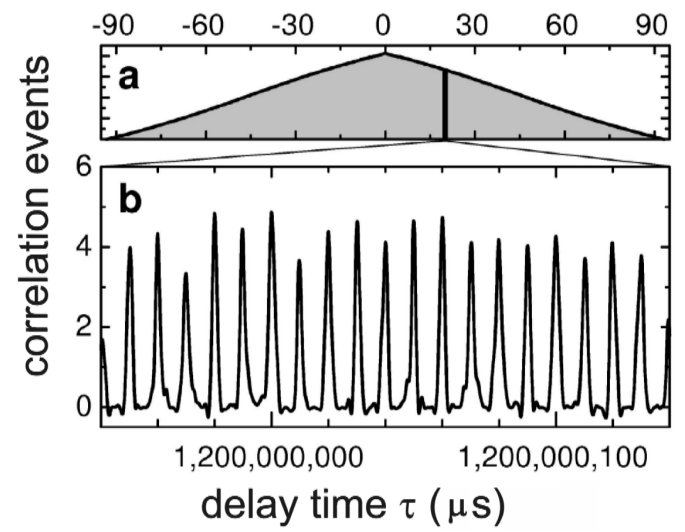

Fig. 1.18 Long-term correlations of photon arrival times at the two detectors. a) Envelope of two-photon correlations recorded over the entire sampling interval, demonstrating the continuous emission of single photons in our system. b) Zooming in at a delay of $\tau=20 \mathrm{~min}$ reveals individual peaks as in Fig. 1.19 for $\tau \neq 0$.

Fig. 1.17b shows a symmetric Gaussian output pulse generated with a weak Gaussian pump pulse. The arbitrary control of the photon pulse shape in the set-up is limited only by the time constants associated with ion and cavity dynamics. This is apparent from the response of the system to a pump pulse with a square profile (Fig. 1.17c). The trailing edge of the pulse decreases exponentially at the cavity decay rate, whereas the onset of the pulse is delayed by the time required to excite the electronic transition. As an example of a more complex pulse shape, we generated a twin-peaked single-photon pulse (Fig. 1.17d). The photon detection times are distributed over two wellseparated maxima, reflecting the structure of the pump pulse. Spreading a single photon over two distinct time bins may be exploited as a way to encode quantum information in the time domain [142].

Apart from the control of the photon's waveform and the suppression of two-photon events, an important accomplishment for applications in quantum information processing is the continuous, uninterrupted operation of our source, allowing correlations to be measured on very long time scales. Fig. 1.18a shows a measurement of correlations sampled over a time of 90 min. The linear decrease of the envelope of the correlation function is a consequence of the finite duration of the measurement. No individual lines are resolved on the time scale shown, but when an arbitrary point on the time axis is zoomed in, the same peak structure is found as in Fig. 1.19 on either side of the origin. As an example, Fig. 1.18b shows correlations between photons detected $20 \mathrm{~min}$ apart. The operation of the system for $90 \mathrm{~min}$ represents 


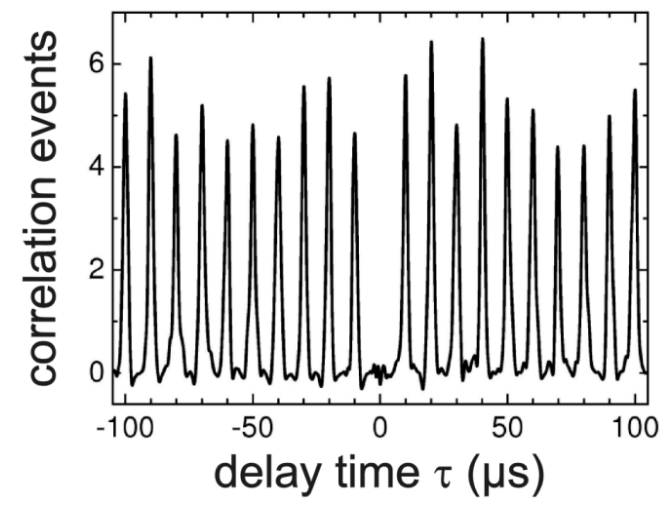

Fig. 1.19 Cross-correlations of photon arrival times at the two detectors (counts in 100-ns time bins) around zero delay. Dark-count events were eliminated with the help of an independent measurement for the data including dark-counts). The absence of a peak at $\tau=0$ confirms that the source emits single photons.

the longest continuous generation of single photons from an atomic source [143]. With ion trapping times of many hours, single-photon emission in the described system can be maintained for even longer periods.

The striking feature of this new single photon source is the fact that it is possible not only to generate a photon on demand or deterministically but also to preselect the duration of the generated photon wavepacket. The latter means that the coherence length of the photon can be preselected. These properties of the single photon source described here will make it a unique source for many applications. The coupling of ions and photons in a controlled way is required in schemes linking optical long-distance quantum communication with iontrap quantum processors, both of which have been successfully demonstrated in the past. The result could be a quantum version of the internet in which local processing sites are connected with each other by photonic channels.

\section{5}

\section{Conclusions and Outlook}

This paper reviews the work on cavity quantum electrodynamics with free atoms. Emphasis is placed on the work performed with the one-atom maser and the ion-trap laser in our laboratory, but a survey on the work done in other laboratories is also given in order to show the large variety of problems being treated with the very fundamental system of an atom interacting with a single mode of a cavity. (For a more detailed review see also [1]).

The experiments on cavity quantum electrodynamics started with studies of free atoms, but meanwhile the field has expanded to include solid-state 
systems, as briefly discussed in the review. Furthermore, new applications are emerging in various directions. First, there are interesting developments of new cavity structures which are leading to new possibilities [144-146]. A further active field is cavity quantum electrodynamics in semiconductors in combination with quantum dots or semiconductor microcavity structures [11, 147]. The latter systems promise application in many areas of optical communication and possibly also in quantum information processing.

The experiments with free atoms will also be further developed. Interesting results may be expected especially in connection with studies of decoherence. The cavity quantum electrodynamic systems with free atoms seem to be the only ones where the phenomenon can be investigated in detail and free of perturbing influences. Furthermore, in connection with the one-atom maser another interesting problem under study is the investigation of the phase diffusion of the maser field.

Phase diffusion of a maser or laser determines the natural linewidth of these systems. In the standard maser/laser, this is caused by spontaneous transitions. In the one-atom maser, spontaneous transitions play no role; there it is the thermal noise which determines the phase diffusion. As this parameter can easily be controlled by changing the cavity temperature, this phenomenon can be nicely investigated. The exploration of phase diffusion is also interesting in the vicinity of trapping states, which were described in Sect. 1.2.5. As shown, the trapping states display the quantum states of the maser field. The behavior of phase diffusion should therefore follow the amplitude-phase uncertainty when the field approaches a trapping state. This phenomenon should be measurable with the available set-up [56].

From the few examples given in this outlook it is obvious that the field of cavity quantum electrodynamics will continue in the future to be as interesting and lively as it has been in the past.

\section{Acknowledgement}

The one atom maser and the trapped ion work is supported by the European Community and the Quantum Information Highway A8 project of the State of Bavaria.

\section{References}

1 H. Walther, B. T. H. Varcoe, B.-G. Englert, and T. Becker, Rep. Prog. Phys. 69 13251382 (2006).
2 E. T. Jaynes and F. W. Cummings, Proc. IEEE 51, 89-109 (1963).

3 D. Meschede, H. Walther, and G. Müller, Phys. Rev. Lett. 54, 551-554 (1985). 
4 M. Brune, J. M. Raimond, P. Goy, L. Davidovich, and S. Haroche, Phys. Rev. Lett. 59, 1899-1902 (1987).

5 J. McKeever, A. Boca, A. D. Boozer, J. R. Buck, and H. J. Kimble, Nature 425, 268-271 (2003).

6 E. Wehner, R. Seno, N. Sterpi, B.G. Englert, and H. Walther, Opt. Commun. 110, 655-669 (1994).

7 G. Nogues, A. Rauschenbeutel, S. Osnaghi, M. Brune, J. M. Raimond, and S. Haroche, Nature (London) 400, 239-242 (1999).

8 A. Imamoglu, D. D. Awschalom, G. Burkard, D. P. DiVincenzo, D. Loss, M. Sherwin, and A. Small, Phys. Rev. Lett. 83, 4204-4207, (1999).

9 M. Sherwin, A. Imamoglu, and T. Montrag, Phys. Rev. A 60, 3508-3514 (1999).

10 G.S. Solomon, M. Pelton, and Y. Yamamoto, Phys. Rev. Lett. 86, 39033906 (2001).

11 Y. Yamamoto, F. Tassone, and H. Cao, Semiconductor Cavity Quantum Electrodynamics, in: Springer Tracs in Modern Physics, Vol. 169 (Springer, Berlin, 2000)

12 M. Oxborrow and A. G. Sinclair, Contemp. Phys. 46, 173-206 (2005).

13 B. T. H. Varcoe, S. Brattke, and H. Walther, Fortschr. Phys. 48, 679-687 (2000).

14 B. T. H. Varcoe, B. Hall, G. Johnson, P. Johnson, W. R. MacGillivray, and M. C. Standage MC, J. Meas. Sci. and Tech. 11, N111-N116 (2000).

15 V. A. Reshetov and I. V. Yevseyev, Laser Phys. 10, 916-922 (2000).

16 M. Orszag, R. Ramirez, J. C. Retamal, and C. Saavedra, Phys. Rev. A 49, 2933-2937 (1994).

17 M. E. Kolobov and F. Haake, Phys. Rev. A 55, 3033-3041 (1997).

18 F. Casagrande, M. Garavaglia, and A. Lulli, Opt. Commun. 151, 395-405 (1998).

19 F. Casagrande, A. Lulli, and S. Ulzega, Phys. Rev. A 60, 133-141 (1999).

20 P. Filipowicz, J. Javanainen, and P. Meystre, Phys. Rev. A 34, 3077-3087 (1986).

21 L. A. Lugiato, M. O. Scully, and H. Walther, Phys. Rev. A 36, 740-743 (1987).
22 P. Meystre and M. III Sargent, Elements of Quantum Optics (Springer-Verlag, Berlin, 1990).

23 M.O. Scully and M.S. Zubairy, Quantum Optics (Cambridge University Press, Cambridge 1997).

24 B.-G. Englert, Elements of Micromaser Physics, preprint, quant-ph/0203052 (1994/2002).

25 U. Fano, Phys. Rev. 72, 26-29 (1947).

26 L. Mandel, Opt. Lett. 4, 205-207 (1979).

27 E. Hagley, X. Maître, G. Nogues, C. Wunderlich, M. Brune, J. M. Raimond, and S. Haroche, Phys. Rev. Lett. 79, 1-5 (1997)

28 X. Maître, E. Hagley, G. Nogues, C. Wunderlich, P. Goy, M. Brune, J. M. Raimond, and S. Haroche, Phys. Rev. Lett. 79, 769-772 (1997).

29 A. Rauschenbeutel, G. Nogues, S. Osnaghi, P. Bertet, M. Brune, J. M. Raimond, and S. Haroche, Science 288, 2024-2028 (2000).

30 B. T. H. Varcoe, S. Brattke, M. Weidinger, and H. Walther, Nature (London) 403, 743-746 (2000).

31 J. H. Eberly, N. B. Narozhny, and J. J. Sanchez-Mondragon, Phys. Rev. Lett. 44, 1323-1326 (1980).

32 G. Rempe, H. Walther, and N. Klein, Phys. Rev. Lett. 58, 353-356 (1987).

33 P. Elmfors, B. Lautrup, and B.S. Skagerstam, Phys. Rev. A 54, 5171-5192 (1996).

34 M. Brune, F. Schmidt-Kaler, A. Maali, J. Dreyer, E. Hagley, J. M. Raimond, and S. Haroche, Phys. Rev. Lett. 76, 1800-1803 (1996).

35 B.-J. Briegel, B.-G. Englert, N. Sterpi, and H. Walther, Phys. Rev. A. 49, 2962-2985 (1994).

36 G. Rempe, F. Schmidt-Kaler, and H. Walther, Phys. Rev. Lett. 64, 2783-2786 (1990).

37 G. Rempe and H. Walther, Phys. Rev. A 42, 1650-1655 (1990).

38 P. Meystre, Cavity Quantum Optics and the Quantum Measurement Process, in: Progress in Optics, vol. 30, edited by E. Wolf (North Holland, Amsterdam, 1992), pp. 261-355. 
39 O. Benson, G. Raithel, and H. Walther, Phys. Rev. Lett. 72, 3506-3509 (1994).

40 G. Raithel, O. Benson, and H. Walther, Phys. Rev. Lett. 75, 3446-3449 (1995).

41 S. Brattke, B.-G. Englert, B. T. H. Varcoe, and H. Walther, J. Mod. Opt. 47, 28572867 (2000)

42 C. Bracher, private communication (2000)

43 H.-J. Briegel and B.-G. Englert, Phys. Rev. A 47, 3311-3329 (1993).

44 B.-G. Englert, M. Löffler, O. Benson, B. T. H. Varcoe, M. Weidinger, and H. Walther, Fortschr. Phys. 46, 897-926 (1998).

45 P. Meystre, G. Rempe and H. Walther, Opt. Lett. 13, 1078-1080 (1988).

46 M. Weidinger, B. T. H. Varcoe, R. Heerlein and H. Walther, Phys. Rev. Lett. 82, 37953798 (1999)

47 S.-Y. Zhu, L. Z. Wang and H. Fearn, Phys. Rev. A. 44, 737-746 (1991).

48 B. T. H. Varcoe, S. Brattke, and H. Walther, New J. Phys. 6, 97 (2004)

49 S. Brattke, B. T. H. Varcoe, and H. Walther, Phys. Rev. Lett. 86, 3534-3537 (2001).

50 S. Brattke, B. T. H. Varcoe, and H. Walther, Opt. Express 8, 131-144 (2001)

51 G. Raithel, C. Wagner, H. Walther, L. M. Narducci, and M. O. Scully, The Micromaser: A Proving Ground for Quantum Physics," in: Supplement 2 to Advances in Atomic, Molecular and Optical Physics, edited by Paul R. Berman (Academic, New York, 1994), pp. 57-121.

52 H. Wiseman and G. Milburn, Phys. Rev. Lett. 70, 548-551 (1993).

53 J. Krause, M. O. Scully, and H. Walther, Phys. Rev. A 36, 4547-4550 (1987).

54 P. J. Bardoff, E. Mayr, and W. P. Schleich, Phys. Rev. A 51, 4963-4966 (1995).

55 C. K. Hong and L. Mandel, Phys. Rev. Lett. 56, 58-60 (1986)

56 M. O. Scully, H. Walther, G.S. Agarwal, Tran Quang, and W. Schleich, Phys. Rev. A 44, 5992-5996 (1991).

57 M. O. Scully and W. E. Lamb, Jr., Phys. Rev. 159, 208-226 (1967); see especially M. Sargent, M. O. Scully, and W. E. Lamb, Laser Physics (Addison-Wesley, Reading, MA, 1974) p. 292

58 Tran Quang, G. S. Agarwal, J. Bergou, M. O. Scully, H. Walther, K. Vogel, and
W. P. Schleich, Phys. Rev. A 48, 803-812 (1993).

59 K. Vogel, W. P. Schleich, M. O. Scully, and H. Walther, Phys. Rev. A 48, 813-817 (1993).

60 C. T. Bodendorf, G. Antesberger, M.S. Kim, and H. Walther, Phys. Rev. A 57, 1371-1378 (1998).

61 M.S. Kim, G. Antesberger, C. T. Bodendorf, and H. Walther, Phys. Rev. A 58, R65-R68 (1998).

62 F. Casagrande, A. Ferraro, A. Lulli, and R. Bonifacio, Phys. Rev. Lett. 90, 183601 (2003).

63 A. Rauschenbeutel, G. Nogues, S. Osnaghi, P. Bertet, M. Brune, J. M. Raimond, and S. Haroche, Phys. Rev. Lett. 83, 5166-5169 (1999).

64 N. F. Ramsey, Molecular Beams (Oxford University Press, New York, 1956).

65 M. Brune et al., Phys. Rev. Lett. 72, 33393342 (1994).

66 J. M. Raimond, M. Brune, and S. Haroche, Rev. Mod. Phys. 73, 565-582 (2001).

67 T. Meunier, S. Gleyzes, P. Maioli, A. Auffeves, G. Nogues, M. Brune, J. M. Raimond, and S. Haroche, Phys. Rev. Lett. 94, 010401 (2005).

68 G. Morigi, E. Solano, B.-G. Englert, and H. Walther, Phys. Rev. A 65, 040102 (2002).

69 B.-G. Englert and H. Walther, Opt. Commun. 179, 283-288 (2000).

70 M. Löffler, B.-G. Englert, and H. Walther, Appl. Phys. B 63, 511-516 (1996).

71 B.-G. Englert, N. Sterpi, and H. Walther, Opt. Commun. 100, 526-535 (1993).

72 P. Maioli, T. Meunier, S. Gleyzes, A. Auffeves, G. Nogues, M. Brune, J. M. Raimond, and S. Haroche, Phys. Rev. Lett. 94, 113601 (2005)

73 J. M. Raimond, T. Meunier, P. Bertet, S. Gleyzes, P. Maioli, A. Auffeves, G. Nogues, M. Brune, and S. Haroche, J. Phys. B: At. Mol. Opt. Phys 38, S535-S550 (2005).

74 P. Bertet, A. Auffeves, P. Maioli, S. Osnaghi, T. Meunier, M. Brune, J. M. Raimond, and S. Haroche, Phys. Rev. Lett. 89, 200402 (2002).

75 P. Lougovski, E. Solano, Z. M. Zhang, H. Walther, H. Mach, and W. P. Schleich, Phys. Rev. Lett. 91, 010401 (2003). 


\section{$42 \mid 1$}

The Deterministic Generation of Photons

76 M. Brune, E. Hagley, J. Dreyer, X. Maître, A. Maali, C. Wunderlich, J. M. Raimond and S. Haroche, Phys. Rev. Lett. 72, 48874890 (1996)

77 R. Miller, T. E. Northup, K. M. Birnbaum, A. Boca, A. D. Boozer, and H. J. Kimble, J. Phys. B: At. Mol. Opt. Phys. 38, S551S565 (2005).

78 R. J. Thompson, G. Rempe, and H. J. Kimble, Phys. Rev. Lett. 68, 11321135 (1992)

79 J. J. Childs, K. An, M. S. Otteson, R. R. Dasari, and M.S. Feld, Phys. Rev. Lett. 77, 2901-2904 (1996).

80 H. Mabuchi, Q. A. Turchette, M.S. Chapman, and H. J. Kimble, Opt. Lett. 21, 1393-1395 (1996).

81 Y. Mu and C. Savage, Phys. Rev. A 49, 5944-5954 (1992).

82 T. Pellizzarri and H. Ritsch, Phys. Rev. Lett. 72, 3973-3976 (1994).

83 P. Horak, K. M. Gheri, and H. Ritsch, Phys. Rev. A 51, 3257-3266 (1995).

84 G. M. Meyer and H.-J. Briegel, Phys. Rev. A 58, 3210-3220 (1998).

85 B. Jones, S. Ghose, J. P. Clemens, P. R. Rice, and L. M. Pedrotti, Phys. Rev. A 60, 32673275 (1999).

86 S. Ya. Kilin and T. B. Karlovich, JETP 95, 805-819 (2002).

87 C. Ginzel, H.-J. Briegel, U. Martini, B.G. Englert, and A. Schenzle, Phys. Rev. A 48, 732-738 (1993)

88 G. M. Meyer, H.-J. Briegel, and H. Walther, Eur. Phys. Lett. 37, 317-322 (1997).

89 M. Löffler, G. M. Meyer, and H. Walther, Phys. Rev. A 55, 3923-3930 (1997).

90 G. M. Meyer, M. Löffler, and H. Walther, Phys. Rev. A 56, 1099-1102 (1997).

91 K. An, J. J. Childs, R. R. Dasari, and M.S. Feld, Phys. Rev. Lett. 73, 3375-3378 (1994).

92 A. D. Boozer, A. Boca, J. R. Buck, J. McKeever, and H. J. Kimble, Phys. Rev. A 70, 023814 (2004).

93 S. Haroche, M. Brune, and J. M. Raimond, Eur. Phys. Lett. 14, 19-24 (1991).

94 C. J. Hood, T. W. Lynn, A. C. Doherty, A.S. Parkins, and H. J. Kimble, Science 287, 1447-1453 (2000).
95 P. H. W. Pinkse, T. Fischer, P. Maunz, and G. Rempe, Nature (London) 404, 365-368 (2000).

96 G. Rempe, Phys. World 13(12), 37-42 (2000).

97 P. Münstermann, T. Fischer, P. Maunz, P. W. H. Pinkse, and G. Rempe, Phys. Rev. Lett. 84, 4068-4071 (2000).

98 A. C. Doherty, T. W. Lynn, C. J. Hood, and H. J. Kimble, Phys. Rev. A 63, 013401 (2000).

99 M. Hennrich, T. Legero, A. Kuhn, and G. Rempe, Phys. Rev. Lett. 85, 4872-4875 (2000).

100 P.W. H. Pinkse, T. Fischer, P. Maunz, T. Puppe, and G. Rempe, J. Mod. Opt. 47, 2769-2787 (2000).

101 T. Fischer, P. Maunz, T. Puppe, P. H.W. Pinkse, and G. Rempe, New J. Phys. 3, 11.1-11.20 (2001).

102 P. Horak, H. Ritsch, T. Fischer, P. Maunz, T. Puppe, P. H.W. Pinkse, and G. Rempe, Phys. Rev. Lett. 88, 043601 (2002).

103 T. Fischer, P. Maunz, P. H. W. Pinkse, T. Puppe, and G. Rempe, Phys. Rev. Lett. 88, 163002 (2002).

104 J. McKeever, J. R. Buck, A. D. Boozer, A. Kuzmich, H.-C. Nägerl, D. M. StamperKurn, and H. J. Kimble, Phys. Rev. Lett. 90, 133602 (2003).

105 J. A. Sauer, K. M. Fortier, M. S. Chang, C. D. Hamley, and M.S. Chapman, Phys Rev. A 69, 051804 (2004).

106 P. Maunz, T. Puppe, I. Schuster, N. Syassen, P. W. H. Pinkse, and G. Rempe, Nature (London) 428, 50-52 (2004).

107 J. Ye, D. W. Vernooy, and H. J. Kimble, Phys. Rev. Lett. 83, 4987-4990 (1999).

108 S. Kuhr, W. Alt, D. Schrader, M. Müller, V. Gomer, and D. Meschede, Science 293, 278-280 (2001).

109 J. McKeever, A. Boca, A. D. Boozer, R. Miller, J. R. Buck, A. Kuzmich, and H. J. Kimble, Science 303, 1992-1994 (2004).

110 J. McKeever, A. Boca, A. D. Boozer, J. R. Buck, and H. J. Kimble, Nature (London) 425, 268-271 (2003).

111 S. Nussmann, K. Murr, M. Hijlkema, B. Weber, A. Kuhn, and G. Rempe, Nature Physics 1, 122-126 (2005).

112 S. Nussmann, M. Hijlkema, B. Weber, F. Rohde, G. Rempe, and A. Kuhn, Phys. Rev. Lett. 95, 173602 (2005). 
113 C. J. Hood, T. Lynn, M. Chapman, and H. J. Kimble, Phys. Rev. Lett. 80, 41574160 (1998)

114 P. Maunz, T. Puppe, I. Schuster, N. Syassen, P. W. H. Pinkse, and G. Rempe, Preprint quant-ph/045136 (2004).

115 A. Boca, R. Miller, K. M. Birnbaum, A. D. Boozer, J. McKeever, and H. J. Kimble, Phys. Rev. Lett. 93, 233603 (2004).

116 C. H. Bennett and G. Brassard, in: Proc. IEEE Int. Conf. on Computers, Systems, and Signal Processing (Bangalore, India) (IEEE, New York, 1984), pp. 175-179.

117 E. Knill, R. Laflamme, and G. J. Milburn, Nature (London) 409, 46-52 (2001).

118 F. Diedrich and H. Walther, Phys. Rev. Lett. 58, 203-206 (1987).

119 T. Basché, W. E. Moerner, M. Orrit, and H. Talon, Phys. Rev. Lett. 69, 1516-1519 (1992).

120 B. Lounis and W. E. Moerner, Nature (London) 407, 491-493 (2000).

121 C. Kurtsiefer, S. Mayer, P. Zarda, and H. Weinfurter, Phys. Rev. Lett. 85, 290293 (2000).

122 R. Brouri, A. Beveratos, J.-P. Poizat, and P. Grangier, Opt. Lett. 25, 1294-1296 (2000).

123 P. Michler et al. Science 290, 2282-2286 (2000).

124 C. Santori, M. Pelton, G. Solomon, Y. Dale, and Y. Yamamoto, Phys. Rev. Lett. 86, 1502-1505 (2001).

125 E. Moreau et al., Appl. Phys. Lett. 79, 2865-2867 (2001)

126 Z. L. Yuan et al. Science 295, 102-105 (2002).

127 C. Santori, D. Fattal, C. J. Vučković, G. Solomon, and Y. Yamamoto, Nature (London) 419, 594-597 (2002).

128 J. I. Cirac, P. Zoller, H. J. Kimble, and H. Mabuchi, Phys. Rev. Lett. 78, 32213224 (1997).

129 A.S. Parkins, P. Marte, P. Zoller, and H. J. Kimble, Phys. Rev. Lett. 71, 30953098 (1993); A. S. Parkins, P. Marte, P. Zoller, O. Carnal, and H. J. Kimble, Phys. Rev. A 51, 1578-1596 (1995).

130 W. Lange and H. J. Kimble, Phys. Rev. A 61, 63817 (2000).
131 C. K. Law and J. H. Eberly, Phys. Rev. Lett. 76, 1055-1058 (1996); C. K. Law and H. J. Kimble, J. Mod. Opt. 44, 2067-2074 (1997)

132 A. Kuhn, M. Hennrich, T. Bondo, and G. Rempe, Appl. Phys. B 69, 373-377 (1999).

133 For a review, see K. Bergmann and B. W. Shore, in: Molecular Dynamics and Stimulated Emission Pumping, edited by H. L. Dai and R. W. Field (World Scientific, Singapore, 1995) pp. 315-373.

134 A. Kuhn, S. Steuerwald, and K. Bergmann, Eur. Phys. J. D 1, 57-70 (1998).

135 A. Kuhn, M. Hennrich, and G. Rempe, Phys. Rev. Lett. 89, 067901 (2002).

136 A. M. Smith and C. W. Gardiner, Phys. Rev. A 41, 2730-2748 (1990).

137 G. S. Agarwal and S. D. Gupta, Phys. Rev. A 42, 1737-1741 (1990).

138 G. M. Meyer, Der Ionenfallenlaser: Einfluß von Dissipation und Pumpdynamik in mikroskopischen Vielniveausystemen, MPQ report 221 (Garching, 1997).

139 M. Löffler, Korrelationen und Bewegungsquantisierung im Ein-AtomMaser und -Laser, MPQ report 235 (Garching, 1998).

140 G. R. Guthöhrlein, M. Keller, K. Hayasaka, W. Lange, and $\mathrm{H}$. Walther, Nature (London) 414, 49-51 (2001)

141 M. Keller, B. Lange, K. Hayasaka, W. Lange, and $\mathrm{H}$. Walther, Nature (London) 431, 1075-1078 (2004).

142 J. Brendel, W. Tittel, H. Zbinden, and N. Gisin, Phys. Rev. Lett. 82, 2594-2597 (1999).

143 L.-M. Duan, A. Kuzmich, and H. J. Kimble, Phys. Rev. A 67, 032305 (2003).

144 see e. g. R. K. Chang and A. J. Campillo, Optical Processes in Microcavities, in: Advanced Series in Applied Physics, Vol. 3 (World Scientific, 1996).

145 K. Vahala, Optical Microcavities, in: Advanced Series in Applied Physics, Vol. 5 (World Scientific, 2004).

146 J. Rarity and C. Weisbuch, Microcavities and Photonic Bandgaps (Kluwer Academic Publishers, 1986).

147 H. Deveaud-Pledran, The Physics of Semiconductor Microcavities (WileyVCH, New York, 2006). 
